\title{
Review of Computer \\ Networking Technology
}

U.S. PARTMENT

OF OMMERCE

National Bureau of Standards

15753

1974 .804 I f 
The National Bureau of Standards ${ }^{1}$ was established by an act of Congress March 3, 1901. The Bureau's overall goal is to strengthen and advance the Nation's science and technology and facilitate their effective application for public benefit. To this end, the Bureau conducts research and provides: (1) a basis for the Nation's physical measurement system, (2) scientific and technological services for industry and government, (3) a technical basis for equity in trade, and (4) technical services to promote public safety. The Bureau consists of the Institute for Basic Standards, the Institute for Materials Research, the Institute for Applied Technology, the Institute for Computer Sciences and Technology, and the Office for Information Programs.

THE INSTITUTE FOR BASIC STANDARDS provides the central basis within the United States of a complete and consistent system of physical measurement; coordinates that system with measurement systems of other nations; and furnishes essential services leading to accurate and uniform physical measurements throughout the Nation's scientific community, industry, and commerce. The Institute consists of a Center for Radiation Research, an Office of Measurement Services and the following divisions:

Applied Mathematics - Electricity - Mechanics - Heat - Optical Physics - Nuclear Sciences $^{2}$ - Applied Radiation ${ }^{2}$ - Quantum Electronics ${ }^{3}$ - Electromagnetics ${ }^{3}$ - Time and Frequency ${ }^{3}$ - Laboratory Astrophysics ${ }^{3}$ - Cryogenics 3 .

THE INSTITUTE FOR MATERIALS RESEARCH conducts materials research leading to improved methods of measurement, standards, and data on the properties of well-characterized materials needed by industry, commerce, educational institutions, and Government; provides advisory and research services to other Government agencies; and develops, produces, and distributes standard reference materials. The Institute consists of the Office of Standard Reference Materials and the following divisions:

Analytical Chemistry - Polymers - Metallurgy - Inorganic Materials - Reactor Radiation - Physical Chemistry.

THE INSTITUTE FOR APPLIED TECHNOLOGY provides technical services to promote the use of available technology and to facilitate technological innovation in industry and Government; cooperates with public and private organizations leading to the development of technological standards (including mandatory safety standards), codes and methods of test; and provides technical advice and services to Government agencies upon request. The Institute consists of a Center for Building Technology and the following divisions and offices:

Engineering and Product Standards - Weights and Measures - Invention and Innovation - Product Evaluation Technology - Electronic Technology — Technical Analysis

- Measurement Engineering - Structures, Materials, and Life Safety * Building Environment ${ }^{4}$ - Technical Evaluation and Application ${ }^{4}$ - Fire Technology.

THE INSTITUTE FOR COMPUTER SCIENCES AND TECHNOLOGY conducts research and provides technical services designed to aid Government agencies in improving cost effectiveness in the conduct of their programs through the selection, acquisition, and effective utilization of automatic data processing equipment; and serves as the principal focus within the executive branch for the development of Federal standards for automatic data processing equipment, techniques, and computer languages. The Institute consists of the following divisions:

Computer Services - Systems and Software - Computer Systems Engineering - Information Technology.

THE OFFICE FOR INFORMATION PROGRAMS promotes optimum dissemination and accessibility of scientific information generated within NBS and other agencies of the Federal Government; promotes the development of the National Standard Reference Data System and a system of information analysis centers dealing with the broader aspects of the National Measurement System; provides appropriate services to ensure that the NBS staff has optimum accessibility to the scientific information of the world. The Office consists of the following organizational units:

Office of Standard Reference Data - Office of Information Activities - Office of Technical Publications - Library - Office of International Relations.

\footnotetext{
1. Headquarters and Laboratories at Gaithersburg, Maryland, unless otherwise noted; mailing address Washington, D.C. 20234.

2 Part of the Center for Radiation Research.

3 Located at Boulder, Colorado 80302 .

- Part of the Center for Building Technology.
} 


\section{Review of Computer Networking Technology}

National Bureau of Standards

Robert P. Blanc

A. $R$ : 9979

Computer Systems Engineering Division

Institute for Computer Science and Technology

National Bureau of Standards

Washington, D.C. 20234

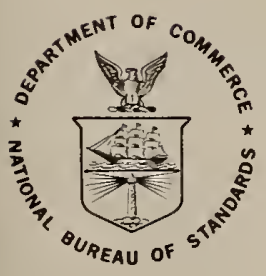

U.S. DEPARTMENT OF COMMERCE, Frederick B. Dent, Secrefary

NATIONAL BUREAU OF STANDARDS, Richard W. Roberts, Director

Issued January 1974 
National Bureau of Standards Technical Note 804

Nat. Bur. Stand. (U.S.), Tech. Note 804, 135 pages (Jan. 1974)

CODEN: NBTNAE

U.S. GOVERNMENT PRINTING OFFICE

WASHINGTON: 1974

For sale by the Superintendent of Documents, U.S. Government Printing Office, Washington, D.C. 20402 (Order by SD Catalog No. C13.46:804). Price $\$ 1.55$ 
This report is one of a series of publications produced by the Institute for Computer Sciences and Technology, National Bureau of Standards, under Grant AG-350 from the National Science Foundation.

This grant supports a broad program of investigation into the foundations of computer networking in support of scientific and related educational efforts.

A listing of completed and planned publications produced by the Institute under this grant follows:

1. Primary Issues in User Needs

D. W. Fife

Chapter 10 in Networks for Research and Education:

Sharing of Computer and Information Resources Nationwide MIT Press, Cambridge, Mass.

Expected Publication January 1973

2. Some Technical Considerations for Improved Service to Computer Users

T. N. Pyke, Jr. COMPCON, 1973

Seventh Annual IEEE Computer Society International Conference

3. Computer Networking Technology - A State of the Art Review

T. N. Pyke, Jr. and R. P. Blanc

COMPUTER Magazine

Computer Society of the IEEE August , 1973

4. Review of Network Management Problems and Issues A. J. Neumann

NBS Technical Note 795, October 1973 
5. Annotated Bibliography of the Literature on Resource Sharing Computer Networks

R. P. Blanc, I. W. Cotton, T. N. Pyke, Jr., and S.W. Watkins

September, 1973

NBS Special Publication 384

6. Network Management Survey.

I.W. Cotton

NBS Technical Note 805 , expected publication December, 1973

7. User Procedures Standarization for Network Access

A. J. Neuman

NBS Technical Note 799 , October, 1973

8. Review of Computer Networking Technology

R. P. Blanc

NBS Technical Note 804, December, 1973

9. Microeconomics and the Market for Computer Services

I.W. Cotton

Submitted for publication

10. Cost Analyses for Computer Communications

R. P. Blanc

NBS Report, expected publication December, 1973

11. Network User Information Support

A. J. Neumann

NBS Technical Note 802 , expected publication December, 1973

12. Quality Service Assurance Experiments

R. Stillman

NBS Technical Note 800 , expected publication December, 1973

13. A Guide to Networking Terminology

A. J. Neumann

NBS Technical Note 803 , expected publication December, 1973

14. Research Considerations in Computer Networking D. W. Fife

NBS Technical Note 801 , expected publication December 1973 
Page

1. INTRODUCTION . . . . . . . . . . . . . . 1

2. FUNCTIONAL CAPABILITIES OF THE COMMUNICATIONS

NETWORK. . . . . . . . . . . . . . . 6

2.1 Interactive . . . . . . . . . . 6

2.2 Remote Job Entry . . . . . . . . . . 15

2.3 Minicomputer-based Systems . . . . . . 19

2.4 Computer-to-Computer . . . . . . . 22

3. TEChNOlogical Features . . . . . . . . . 26

3.1 Communications Circuits . . . . . . 26

3.2 Communications Hardware . . . . . . . 31

3.3 Communications Software . . . . . . . 36

3.4 Configuration . . . . . . . . . . . 41

4. EXISTING NETWORKS . . . . . . . . . . . 45

4.1 TYMNET. . . . . . . . . . . . . . 46

4.2 CYBERNET . . . . . . . . . . . . . 59

4.3 GE Information Services . . . . . . . 70

4.4 MERIT . . . . . . . . . . . . 81

4.5 ARPANET . . . . . . . . . . . . 91

4.6 TSS . . . . . . . . . . . . . 104

5. CONCLUSIONS . . . . . . . . . . . . 112

REFERENCES . . . . . . . . . . . . 125 

Review of Computer Networking Technology*

Robert P. Blanc

This report gives a descriptive summary of the technical characteristics of existing computer networks, including data communication technology and configuration related to support of resource sharing services for a computer network. Included are discussions of terminal support capabilities for the communications network and a development of relevant network terminology. The report concludes with a comparative evaluation of existing technological approaches to networking.

Key words: Computer networks; computer-to-computer transfers; interactive terminals; minicomputer-based systems; network configuration; remote job entry; resource sharing.

\section{INTRODUCTION}

A review of the technical characteristics of existing approaches to computer networking is presented for the purpose of comparative evaluation in relation to their potential for supporting resource sharing for the Networking for Science Program of the National Science Foundation [1]. For the purpose of this review, a computer network is considered to be any interconnection of an assemb1y of computer systems and/or terminals together with communications facilities [2]. Resource sharing in a computer network refers to the sharing among network participants of computer resources, including hardware, software, and data files. The purpose is to make available, nationally, special computer resources which are not locally accessible, such as processors unique in speed or storage capabilities, specialized programs of interest to 
a distributed clientele, and large data files too expensive to reproduce. The coverage in this review is of sufficient depth but general in scope so that it should be useful to those planning, implementing, evaluating, and using other resource sharing computer networks. It is impractical to examine each technical detail of a network and relate it directly to resource sharing, since such a one-to-one correspondence is probably non-existent. But the features of a network will be described and, in whole, related to a set of network support requirements which are themselves prerequisites for sharing computer network resources. The categorization of the requirements will be by terminal or communications support, not by the end service provided, such as program preparation or information retrieval. The purpose in discussing these communications support functions is to identify techniques for making the user interface to existing networks more flexible and effective and to provide services that do not presently exist.

These support functions, which will be discussed in the first part of the paper, are the capabilities of supporting various classes of computer-based service, including interactive or conversational, remote job entry, where input and output are performed with card readers and 1 ine printers respectively, the support of minicomputer based systems such as automated labs, and computer-to-computer communications. Some of these capabilities are considered more essential 
than others and this will be clarified in the text.

The next section is devoted to the description of the technical characteristics which can be used to classify a network. These attributes will be described, and alternatives in implementation, compared. Advantages and disadvantages for application in the Networking for Science Program are presented where possible. Of course, in most cases, it will not be clear whether a particular feature by itself is advantageous. Rather, it is the combination of technical attributes in the implementation of a network that can be evaluated. A byproduct of these discussions is the development of a working terminology for the technological aspects in computer networks.

The various technological approaches to networking in several selected existing networks are then examined. Completeness has not been achieved, but a substantial number of networks will be considered as representative of the available approaches to network technology. These networks include: TYMNET, the commercial timesharing network of Tymshare, Inc.; CYBERNET, the Control Data Corporation commercial network; GE Information Systems, the General Electric Corporation commercial network; MERIT, the Michigan Universitys' network; ARPANET, the experimental network of the Department of Defense Advanced Research Projects Agency; and TSS, an experimental network of IBM $360 / 67$ Time-Sharing System computers. This report is confined to existing 
approaches, and while suggested improvements in those approaches may be indicated, there will be no attempt to specify a new, but non-existent approach to network technology. A discussion of a network technology employing only off-the-shelf concentrators and multiplexers may be important for cost comparison, but is not addressed by itself in this report. The technical aspects of such an approach are wellunderstood and in addition, its application to a network of distributed heterogeneous computers is inconvenient at best. Concentrators and multiplexers will, however, be considered as specific network components, and at least one network making extensive use of concentrators, along with more sophisticated switching computers, will be discussed at length.

Each technological approach will be discussed in terms of technological characteristics and their ability to support the functional requirements. In addition, each of the networks possesses a number of salient features which will also be identified. The information presented in this section was obtained through personal contact with principals of the networks and/or through extensive investigation of the literature including that referenced in the annotated bibliography on resource sharing computer networks [3].

The last section evaluates the alternative approaches indicating the desirable features and issues related to the requirements of the Networking for Science Program. Although 
it is difficult to separate technical and economic concerns, this review concentrates on technical factors - desirable technical characteristics and constraints of the technology because of various mixes of performance requirements.

It is important to note that, although specific networks are identified, there is not intent to favor or criticize those particular networks. Rather these networks have been chosen as well-known representations of important approaches to networking technology and it is the review of those technologies that is the purpose of this report.

NOTE: The identification of certain commercial equipment and networks in this report is done in order to adequately identify the network components and technologies discussed herein, and in no sense does it imply recommendation or endorsement by the National Bureau of Standards. 
2. FUNCTIONAL CAPABILITIES OF THE COMMUNICATIONS NETWORK Alternative technologies should be evaluated in terms of their responsiveness to user needs. One approach is to identify those needs and, subsequently select a communications technology to satisfy them. This approach is iterative in nature; that is, the best available data concerning user needs is employed in evaluating different alternatives, and as the data improves, so will the evaluation. At this point in time, a number of basic requirements can be identified in terms of particular types of usage that the communications network should be capable of supporting. These types of usage to be considered are interactive, remote job entry, communications with minicomputer based systems, and computer-to-computer transfers.

\subsection{Interactive}

Interactive uses depend upon the use of teletypewriter or equivalent terminals to communicate with a remote computer system. Computer terminals are the mechanisms by which information stored in computer data banks becomes translated into action by way of human beings. Of importance in evaluating a communications network are the habits of the terminal users, the characteristics of the terminals themselves, and the constraints placed on the communications network by these terminals.

Code, speed, and mode of operation are important characteristics of terminals directly related to the communications 
facility. There does exist a standard code for information interchange, ASCII, as specified in Federal Information Processing Standards (FIPS) Nos. 1 and 15, and this code should be supported by the network. For systems to be a part of a Federal installation, this support is in fact a requirement. In general a network must impose a series of constraints to operate effectively, and in the case of terminal to computer communications, the standard code is a constraint which is beneficial in that it diminishes the time and money spent on interfacing terminals, a cost ultimately borne by the user. Later when we examine the ARPANET we will see that its terminal interface is capable of supporting non-standard devices. This may at first appear desirable, but consider the scarce resource, main memory, that must be devoted to the variety of non-standard devices. In the ARPANET example, over onethousand (1000) words of memory of the twenty-eight thousand $(28,000)$ word minicomputer terminal interface are dedicated to software and tables to interpret the code of the non-ASCII devices [2]. It has been suggested that users of non-standard terminals pay an additional charge for the added resources that they consume, and this would appear to be one reasonable alternative.

Speed of terminals can range from 110 baud for a simple teletype to 9600 baud and greater for a C.R.T. Terminal with vector capability. Many communications networks are restricted to speeds considerably lower than that maximum 
on the high capacity outgoing channel (that connecting the remote concentrator to the computer or computer front end) and are even more restricted on the slow speed input channel (that connecting the terminal to the concentrator or multiplexor) and are therefore incapable of supporting the higher speed devices.

Speed recognition, the automatic identification of terminal speed by the network, although not a basic requirement, is another capability worth considering in evaluating technologies to support interactive usage. When the terminal interface servicing dialup ports is capable of supporting a mix of speeds without devoting particular ports to particular speed devices, it will statistically provide better service in terms of the probability of obtaining a free port for attaching a device to the interface. That is, it can be shown that the probability of obtaining a free port is greater if a11 ports are accessible to any speed device than if subsets of the ports are devoted to particular speed devices. The physical realities of the two situations are: in the first case a user can dial a single number regardless of the speed of his device to obtain access to any port (ports are on a rotary) which will adapt to his device speed through automatic speed recognition; or for the latter situation, the user will dial, for example, three different numbers for three different devices to obtain access to any of a subset of ports (the subset is also on a rotary). 
The modes of operation of a terminal, which for many terminals is switchable, can be full-duplex or half-duplex transmission depending on the terminal itself, and the computer system on the network with which the terminal is communicating. Half-duplex refers to the case where the user's keyboard is connected to the printer at the terminal, and to the convention that when the computer is producing output it does not respond to input from the user (except perhaps for a special "break" signa1). When the computer is not typing it is in a mode to accept input from the user [4]. Fu11-duplex refers to the case where the user's keyboard is locally disconnected from the printer mechanism. When characters are received at the computer, they are returned to the user. The returned character or characters may not be identical to the received character; for example, some systems will return a control character as an up-arrow followed by the character ("ETX" is sometimes returned as "^c"). The process of sending the received characters, exactly or modified, is often referred to as the echo-plex mode of operation. Full-duplex, however, also implies the separation of input and output to the point where a user at a terminal can be typing input while the computer is sending him output. In this case "echoing" will be delayed until the output has been completed. It is not unusual, nor particularly distasteful, to hear the terms fullduplex and echo-plex used interchangeably although it 
is preferable to think of echo-plex as a subset of fullduplex (that subset related to the actual echoing of characters).

The communications network must be capable of fielding these alternatives. For example, if echoing is desired in the ful1-duplex mode, then in the network it must be determined which communications component is to perform the echoing and when. Some networks allow the interactive user to set and alter the mode as desired, while others are far less flexible. As will be seen in later discussions, the proper control of echoing is important to the efficient use of a network.

Line utilization generated by interactive usage is another consideration relevant to communications technology. Models together with actual data, for example, that derived by Jackson, Stubbs, and Fuchs of Bel1 Telephone Laboratories, can be used to predict communication facility utilization as a func tion of the number of interactive users, their application, and the speed of their terminals $[5,6]$.

The "data stream" model derived is used to study the characteristics of holding time, defined as the time from connect to disconnect between a terminal and a computer system. However, the model has general applicability in the analysis of communication facility utilization and the tailoring of a communications system to the characteristics of interactive users and computer systems. Using the "data stream" 
model it was found that system load can greatly effect holding times, that user delay or think time is a significant component of holding time, that user send time is insignificant, and that computer transmit time is the smallest component of holding time. So the model provides a tool for determining the percentage of holding time during which useful work is performed and a means of studying ways to adapt communications systems to terminal traffic characteristics.

Statistics are also available from several networks in terms of the average number of characters generated per second by interactive usages at specific data rates and the number of interactive users that can be supported on a communications link of a specified capacity. Specifically, it was learned that on the TYMNET network using interactive users in the speed range of 110 to 300 baud, the average traffic generated by a user including both sending and receiving is 5.5 characters per second; and it is possible to support forty-six (46) interactive users over a 2400 baud communications circuit. Similar figures for the GE network indicate terminal duty factors based primarily on terminal output statistics to be 7 characters per second for 110 baud terminals [7].

Using the model of Jackson and Stubbs, the figures from GE and TYMNET and the preliminary data of a Network Measurement Machine [4] developed at the National Bureau of Standards, the following tabulation has been generated: 
Average Characters Per Second (Half Duplex)

Max User System Combined

Network Measurement Machine

(applied to scientific users)

10

2.3

7.5

4.5

TYMNET

10

0.5

5.0

GE

7

30

21

Jacks on, Stubbs

(moderately loaded scientific)

10

0.48

8.8

3.6

(heavily load scientific)

10

0.27

2.1

1.5

(moderately loaded business)

15

0.8

8.3

4.0

In the above the maximum utilization "max", refers to the channel capacity. The channel is composed of modems, circuits, interfaces, and terminals, and its capacity is determined by the most limiting component. In the preceding, the terminal speed of 110 baud determines the channel capacity; because the commonly used modems, 103 A's or equivalent, are capable of 300 baud and voice grade circuits are capable of operating at even higher speeds. The column labeled "user" indicates the average number of characters generated per second when the user has the channel. Typically this will be during the period of user think time combined with user transmit time. The column labeled "system" refers to the average number of characters per second generated during the time at which the system has the channel. This time will be the sum of response time and transmit time. The "combined" column is the average weighted by time. 
This kind of data is essential for the analysis of the relationship in terms of loading between the communications network and the interactive user. In many networks, a concentrator is used to interface interactive terminals on its low speed side to the network on its high speed side. Assuming that the bandwidth of the minicomputer used as a concentrator in terms of speed and buffer space will not be the limiting factor, then knowing the average traffic generated by a given speed terminal, and knowing the efficiency of the communications discipline, the number of terminals that can be serviced on a given high speed channel can be calculated. Average traffic statistics are somewhat useful since concentrators employ asynchronous multiplexing; that is, bandwidth under-utilized by some terminals will be utilized by those requiring it rather than devoting fixed time slots to terminals regardless of utilization as is the case with synchronous multiplexing [8]. The averages though can be somewhat misleading in that averaging holds only for large numbers of terminals and the peaking effects are somewhat unpredictable. So the averages are more meaningful for concentrators that support 1 arge numbers of terminals. The efficiency of the communications discipline indicates the amount of actual data that can be transmitted in a message block allowing the overhead. These factors all taken together might give an accurate projection of compatibility between a high speed communications channel and a number of 
interactive terminals for a particular concentrator, but the analysis in fact is somewhat more complex. Most communication facilities are symmetric; that is, input bandwidth equals output bandwidth, while user interactions with a system are typically asymmetric heavily favoring output or traffic from the system to the interactive terminal. Therefore, total average traffic or average number of characters transmitted is not as significant as the average number received by a terminal from a system since traffic in this direction will correspond to the greatest line utilization and is the traffic that the line capacity must match. Further more, these averages taken over a total network with a large number of users and different kinds of terminals and systems are not nearly as useful as burst characteristics relative to particular kinds of uses, known terminal characteristics, and given systems.

A Network Measurement Machine has been developed at the National Bureau of Standards for measuring the activity of interactive users, communications facility utilization, and the performance of a network as seen by its users [4]. This device interfaces at some point between users and systems and, operating in transparency to both system and user, records the entire dialogue, time tagging and identifying as to source each character received. This makes it possible to select networks, systems, mixes of terminals, and types of users described by discipline or application and vary 
whatever parameters are appropriate to arrive at the desired results on traffic characteristics. For a system designer this type of measurement makes it possible to tailor a communications facility to a specific class of users or to vary the mix of users to accommodate an existing communications facility. From the users standpoint, application of the Network Measurement Machine allows a network subscriber to specify performance requirements for a communications network, and to then monitor the network performance to see that the requirements are consistently met. For example, if response time is the criterion it is possible to measure the communication network plus system performance (computer system with which the terminal is interacting) or to measure only the effect of the communications network. This can be done by comparing data collected when the terminal is connected through the network with that obtained when the terminal is connected directly to the computer system bypassing the network. This latter type of measurement should be particularly useful to users of a communications network.

\subsection{Remote Job Entry}

Remote job entry is a computer usage mode employing card readers, line printers, and possibly card punches located remotely to submit jobs and receive output from a computer system. Communication with a remote job entry device will typically require a higher data rate than communication with interactive terminals and synchronous instead of asynchronous 
transmission as in the case of interactive terminals. With asynchronous transmission, each character is initialized by a start bit and terminated by a stop bit adding two bits of overhead to each character transmitted. Synchronous transmission is continuous with regular timing, the bits of one character immediately followed by those of another with no start and stop bits and no pauses, thus giving the most efficient line utilization. Besides being inherently faster because of the elimination of start and stop bits, synchronous transmission uses higher speed modulation techniques than asynchronous transmission; specifically multilevel signaling can be used. Also, synchronous transmission can tolerate a higher degree of distortion on high speed circuits than can asynchronous transmission. In addition to the speed advantage, there is also an advantage in the integrity of the data transmitted. The stream of characters is normally divided into blocks for synchronous transmission. At the end of each block an error-checking pattern is transmitted based on the contents of the block. The receiving system must generate the same pattern or an error is realized. Acknowledgement of good data and timeouts and/or negative acknowledgements for bad are used to control retransmission when necessary. The specifications of the block size, the method of error detection and correction, and the formatting and interpretation of the data transmitted determine a protocol. 
The synchronous communication requires the development of a synchronous protocol to establish the proper two-way exchange of information between the remote job entry device and the communications network. This protocol may vary among devices and therefore requires flexibility in the network to recognize and adjust to different devices, or restrictions as to which devices are allowed on the network. ANSI Standard X3.28-1971 specifies procedures for the use of communications control characters of American National Standard Code for Information Interchange (ASCII) in specified data communication links.

Many existing networks provide adequate support for interactive termina1s, but make no provisions for remote job entry devices in their communications network. This raises an interesting question. Should remote job entry be supported on the same communications facility as interactive terminals? Remote job entry and interactive uses can entail different traffic patterns and quantities, different communications protocols, different buffering requirements in the communications network, different interfaces, and different response requirements. It may be true that the network that most effectively and economically supports interactive terminals is not efficient for remote job entry. On the other hand, the potential "economies of integration" of providing a single facility to handle both kinds of application cannot be ignored, nor can the convenience to the user requiring both 
of finding them on the same network. Therefore both courses should be considered in the evaluation of alternatives.

As will be examined in more detail later, existing networks have taken different approaches to handle this mix of needs. TYMNET is tailored for interactive traffic, and for the moment, ignores remote job entry [9]. CYBERNET supports remote job entry traffic on its wideband links [10]. GE was developed for interactive use with background batch systems accessible through the interactive network [11]. ARPANET will handle both with the same communications network, but it is not clear what the effect on response time is of mixing interactive and remote job entry traffic under conditions of heavy loading [12]. It should be realized that the differences in the device characteristics are customarily resolved at the interface and then transmission is synchronous over the high speed circuits of the communication network. The distinctions to the communications network are those of the different kinds of traffic generated and the different response times required. Interactive users will generate small messages (block size may be constant but the actual data within the block is small in amount) and require rapid, consistent response times of less than two seconds. Remote job entry users may transmit large messages or multi-block messages being interested more in total throughput than in response time to any particular message. There has been a proposal to divide a communications link into two logical channels, one being a demand channel 
for short messages and quick responses, the other being a reservation channel for long messages [13]. The reservation for that channel could be made over the demand channel. This is meant to be particularly applicable to satellite links and presents an interesting concept for continued research.

\subsection{Minicomputer-based Systems}

Minicomputers can communicate with large computer systems for the purpose of providing support to the large systems in the form of front end processing or device control, for example, or the minicomputer can be the receiver of support from the large system where the system provides a service more economically than can be performed in the minicomputer, such as cross-compilation (advantages in speed and editing capability), large computations, and large file storage. In the former, minicomputers can be an integral part of a network, acting as an interface between the user at his terminal or remote job entry device and the network of computers. This supporting role of the minicomputer is very important when discussing network functions in relation to components, but it is the latter interaction - the network supporting the minicomputer - that is relevant to the discussion of network capabilities and customer needs.

Minicomputers can relate to a network in a hierarchy of three levels. At the lowest level there is the minicomputer or intelligent terminal which will be the user of the network analogous to a human user at a terminal. At the second 
level is the interface to the network which quite probably will also be a minicomputer. The interface is analogous to the terminal interface (often a concentrator) in the case of the human user. This interface for the minicomputer-based system (hopefully a single interface can control many minicomputers) cando as little as provide access to the network or can be quite sophisticated both in the services it provides as an interface and the services it can provide locally which will eliminate the need to access the network in some cases. For example, a sophisticated interface could recognize and resolve differences between the minicomputer based systems and it could realize protocols for performing simple tasks with remote systems on the network such as logging in. Furthermore, it could provide simple compilers and file storage to the mini-based systems so that they could perform those tasks without the necessity of even using the network. In both cases the support can occur in varying degrees depending upon the intentions of the designer. At the third level is the network itself with its variety of host computers. Such a hierarchy is presently being implemented at the Institute for Computer Research at the University of Chicago [14]. The minicomputer, which might be monitoring or controlling a laboratory experiment or controlling a number of other minicomputers which are themselves controlling experiments, needs certain services from a larger computer or computers on the network. For example, the minicomputer 
could store its large files on the disk system at a larger remote computer. A larger computer might have a cross-compiler which has the capability of producing code which will run on the minicomputer. Due to the relatively high cost of minicomputer peripherals [15], a number of minicomputers could share a set of peripherals located somewhere on the network. The minicomputer could be sending data to a larger system for the purpose of obtaining large scale computations.

These services are obtained by the minicomputer through interacting with the processes on a larger system much the same way as a human user would. Difficulties arise, however, in that the outputs from these processes are somewhat unpredictable and require responses from the observer. Since the observer is a minicomputer, it must anticipate all system responses, which as stated can be unpredictable. The introduction of a network of computer systems with which the mini can interact to receive services compounds the problem as the proliferation of heterogeneous systems makes responses even less uniform. This indicates that a set of protocols should exist on the network to standardize the interaction between network computer systems and minicomputer based systems that they serve.

A recent paper presents three (3) potential solutions to the problem including : (a) special controlled protocol channels in which a clean, well-behaved channel all the way to the applications program is provided to the automation 
user; (b) special filters at some point between the user and the applications program to assure the automation user a uniform-10oking network; (c) a very smart terminal node that can take into account and react to a wide variety of messages and initialization sequences [16].

The important consideration in evaluating an existing network relative to minicomputer based systems is that it be flexible enough to accommodate the communication needs of the minicomputer and the development of the necessary protocols. For example, sufficient bandwidth in the links of the netwurk connecting the minicomputer to the target computer system must be available to facilitate the traffic, anticipated to be more intense than that of interactive human users. A file transfer capability would be useful, for example in the application of cross-compilation where source code and object modules are exchanged. Synchronous communications between nodes is rertainly important for handling the volume of traffic generated at a satisfactory speed. Therefore, although there may presently exist few networks which satisfactorily support minicomputer based systems, there do exist more networks commensurate with the requirements for development of this support.

\subsection{Computer-to-Computer}

Computer-to-computer communication is comprised of interprocess transfers, where the processes are software processes or programs running on different, separate host computers. 
There are a number of kinds of applications for which this kind of communications may be useful including: data sharing, program sharing, load sharing, and multiprocess solution to a problem. Data sharing is a mode of network operation in which programs or data requests are sent from various computers on the network to utilize a data base resident at a particular node. This is particularly useful when the data base is large and costly to transport or reproduce and the programs are relatively small. When the opposite is true, large programs and relatively small amounts of data, then program sharing may be appropriate. This is a mode of operation in which data is sent to use a program resident at a particular node. Load sharing is a mode of network operation in which a workload is distributed among the service computers on the network to achieve a balanced compute load at each site.

Multi-process computing is a mode of network operation generating true process-to-process communications in which an application requires the execution of programs concurrently on several processors. One of the few examples of this type of operation is the McRoss experiment which simulates an air traffic control system through execution on distributed PDP-10 TENEX systems on the ARPANET [17]. These kinds of applications are certainly intriguing to the software engineer but at this time are a limited utility to the discipline-oriented customer interested in using a network 
to support research, not as the object of research. To allow for the possible incorporation of this form of communication at a future time, if it becomes stabilized and noncumbersome to use, requires a communications network consisting of links of considerable bandwidth to avoid propagation delays and provide rapid interprocess communication between computers, compatible to that internal to a computer. Sophisticated protocols will also have to be developed to facilitate comfortable interaction between computers.

Special capabilities must be implemented to provide for any kind of convenient computer-to-computer communication. For a user to transfer a program from one computer to use a data base at another, for example, there should exist a standard, easy to understand and easy to use calling sequence to trigger the interaction regardless of the systems in use. Where such network control languages have been developed, then computer-to-computer communications is an intriguing possibility for the applifations user. Solving a problem with programs running on distributed processes is certainly more sophisticated than data or program sharing and at this point in time does not constitute a mandatory capability for the present considerations. In any case, for computer-to-computer communications to be effective a substantial degree of reliability among participating systems is necessary when processes are dependent upon al1 of those systems. 
When evaluating existing networks, it becomes clear that some are more capable of accommodating computer-to computer communications than others. It must further be determined if this capability has been developed at an expense considerably greater than that required to satisfy the other capabilities and if its usage results in the deterioration of service for those other functions. 


\section{TECHNOLOGICAL FEATURES}

In order to characterize the technology employed in an existing computer network, it is necessary to consider the features which are the components of the technology. There are those features which can be used to describe the componential structure of the network and the interrelationships of those components, and these will be described under the category of communications. Other characterizations, take a more global view of the network, and these will be described in the discussion on network configurations. Whenever practical it will be shown how a particular feature and the manifestations of that feature relate to the functional capabilities previously discussed. Particular features, when considered in isolation, are not necessarily conducive to analysis of their advantageous employment in a computer network, but rather it is the combined effect with other attributes that allows evaluation. Nevertheless, in some cases it will be possible to make generalizations about the effectiveness of the usage of alternative components. The primary purpose of this section, however, is to identify technological features and clarify the terminology for use in the next section, where existing networks are described and characterized.

\subsection{Communications Circuits}

In a computer network there are those circuits that interconnect the nodes of the network, circuits that connect ter- 
minals with the network, and those circuits, logical or physical, which provide the end-to-end path between communicating processes. Consider first the circuits that physically connect the nodes in a network. The transmission media here can be voice grade lines or wideband and they can be owned, leased from a common carrier, or dialup. Voice grade circuits can be modulated to operate in the range of 110 baud to 9600 baud. Wideband 1 ines operate from 19,000 baud to 250,000 baud and some economies of scale exist if there is in fact a need for that kind of bandwidth. For a more complete discussion, see Reference 2. The functional requirements discussed earlier could potentially be satisfied by voice grade lines, although a lot depends on the topology and traffic patterns of the network, as it might, for example, be more economical to provide a single very high speed link than to provide multiple lower speed lines to accommodate known network traffic [18]. The decision of whether to lease (or purchase) lines or to rely on dialup facilities depends on utilization. First, dialup lines are at present restricted to 4800 baud or less (2000 baud with common carrier provided modems). This could cause an unsatisfactory level of service to those functions performed marginally at that speed, for example, remote job entry utilizing some of the faster and more sophisticated devices. In addition, if holding times are significant then the dialup facility will be uneconomical where toll calls are involved. 
The second kind of circuit in a network is that connecting a customer device - timesharing terminal, remote job entry station, minicomputer based system, etc. - with the network interface, possibly a multiplexer or concentrator. This circuit, part of the low speed channel side of the multiplexer or concentrator, can operate at a maximum speed equal to that of the circuit connecting nodes, but is usually sharing that high speed circuit with a number of other low speed circuits and thus operates at a considerably lesser baud rate. Voice grade facilities are sufficient for most applications. The speed achieved through wideband service can not normally be utilized except, possibly, in a computer-to-computer transfer where one computer is entering the network as a terminal port to interact with a remote computer doing 1 ikewise. Holding times with interactive terminals are usually short enough to justify the use of dialup facilities, although leased lines are sometimes employed in the more critical applications to guarantee access. A11 of these physical circuits can be used as either full-duplex, allowing for twoway simultaneous transmission, or half-duplex, facilitating two-way transmission but in only one direction at a time. This is dependent upon the number of physical wires ( 2 or 4 wire), or upon the modulation techniques used ( 2 wire can be used in fu11-duplex mode) .

Also the circuit can be synchronous or asynchronous, the synchronous interface typically offering higher speeds and 
better error checking at a higher cost. Ideally the network should be capable of supporting both types of circuits for terminal connections.

The third kind of circuit in a computer network can utilize the other circuits in its establishment. This is the end-toend circuit which connects two processes, for example a program in a computer with a user at a terminal or two programs in two different computers. In contrast, those circuits discussed previously connected two or more physical devices and will be used in part to form this circuit, a physical or logical connection between processes. If the circuit is physical, then switching hardware will establish the circuit making the proper connections at each switching point. If the circuit is logical, than no direct physical connection exists at any of the switching points, necessarily computers in this type of communication between the input and output lines, but instead, the connection is established in tables of the communications software of the computers establishing the connection, and is executed by other computers at each switching point. In any case, whether the circuit if physical or logical, it remains in existence for the duration of the interprocess communication. When the circuit is physical, then line or circuit switching is the terminology used to describe the communications protocol. When the circuit is logical, existing terminology does not adequately describe the con- 
dition, but the term "message switching" has been employed. Message switching, however, properly refers to communication without the establishment of. a circuit, or perhaps it would be more accurate to say that the circuit is established only for the duration of a single message.

For discussions in this report, and to remain compatible with similar discussions found in the literature, we will make the following distinctions: line switching establishes a physical circuit path, physically connecting the input path to the appropriate output path at each switching point, and exists for the duration of interaction between processes; virtual circuits are circuits that are established in the tables of the switching computers logically connecting an input port to an output port for the duration of interaction between processes; message switching refers to the absence of a circuit, logical or physical, relying instead on destination addresses found in the message itself so that the switching computers can determine the appropriate path or ports to use for the message to arrive at the known destination For additional clarification line switching is commonly applied to the public switched network accessed through dialing, the virtual circuit concept is used in describing TYMNET [20], and message switching occurs between switching computers in the GE network [11]. In the ARPANET, a special variety of message switching, called packet switching [19], occurs. In this case, messages from a host computer are further divided, by the net- 
work interface message processor, into packets for transmission in the network.

It is not clear which kind of circuit technology is best suited to a generalized network. Arguments exist favoring the logical circuit and message switching concepts for making better use of carrier facilities to increase effective throughput on a given speed circuit and for allowing greater flexibility in interprocess communication as to which process can interact with what other processes over which circuit. This is because many logical circuits can be established or many messages switched with different sources and destinations over a given physical circuit. Although this increased capability employs a more sophisticated technology than line switching and a proportionate degree of complexity in its development and its subsequent use, its importance is gaining increasing acceptance $[11,19,20]$.

\subsection{Communications Hardware}

Communications hardware encompasses devices for transmission, switching, and termination of communications signals, such as data terminals, modems, acoustic couplers, PBX equipment, store-and-forward message switching computers, etc. Many of these, such as modems and acoustic couplers, are not germane to the discussion of existing networks. What is important to the discussion of networks are the interfaces between the host computers and the communications network, the interfaces between the users terminal devices and the communications net- 
work, and the message switching computer. Communications network refers to the network exclusive of the user terminals and host computers, or to paraphrase, those components of the total network connecting the user terminals to the host computers. In ARPANET terminology, the host computer nodes and the communication network are the "resource sharing computer network", while the communications network is the "communications subnet" [19]. Selection of one type of network communications component over another is difficult to evaluate without entertaining an economic argument, but where distinct technical advantages exist relative to functional capabilities they will be presented.

The interface between the host computer and the network can occur in three different ways corresponding to three different hardware considerations. First, the host computer can be an integral part of the communications network, possibly performing a message switching function for the network (where messages will be directed to itself or other nodes on the network), and thus requiring no special interface computer. The IBM research network, Network 440 was configured this way with a central computer acting as a host and a message switcher [21]. Second, the host computer can be connected to the network through the message switching computer, again requiring no additional hardware other than the transmission medium, as is the case with the ARPANET [19]. Last, the computer may be connected to a front-end computer which acts as 
an interface to the message switching computer and therefore the network, intercepting and controlling all input and output between the network and the host computer. The ARPA Network Terminal System (ANTS) could be applied as a front-end computer in this manner [22].

The use of front-end computers has a lot of appeal in networking. It can perform the functions of network interprocess communications control controlling the flow of messages between processes in the host computer and processes elsewhere on the network, and it can be responsible for making and breaking the connections between processes. These functions can be performed in the host computer itself, but with three distinct disadvantages:

(1) extensive revisions and additions to the host system may be required;

(2) substantial overhead in the host computer may be required to perform these functions;

(3) transportability is minimal or non-existant except to computers with identical operating systems.

Furthermore, a front-end computer in addition to acting as a host computer interface can also function as a terminal controller and possibly a peripheral controller. There are however two disadvantages to using the front-end technique:

(1) two controllers may be required--the standard host controller plus the front-end computer; 
(2) the host computer's network interaction is limited to that supported by existing host communications hardware and software.

The interface between the terminal devices and the communications network is usually a multiplexer or concentrator. A multiplexer is a non-programmable device with predetermined speed selections and a concentrator is a programmable computer. Concentrators offer a number of advantages over multiplexers. Multiplexers employ fixed time slots on the high speed link to transmit data from the terminals and/or other devices being multiplexed and cannot take advantage of under utilization of some devices to accommodate increased utilization on others. Concentrators, on the other hand, with their buffering capabi1ity and programmed logic can use the high speed link to the optimal advantage of all devices [8]. Since concentrators are programmable they can perform automatic speed recognition to accommodate automatically different speed devices, whereas multiplexers are limited to a single speed or predetermined set of speeds. Concentration need not be performed on a computer devoted to that application but may be merely a function of a general purpose minicomputer which is also acting as one of the switching computers in a network such as is done in the TYMNET [20] and ARPANET [23]. One seemingly practical approach is to use concentrators as network nodes and use multiplexers to access the concentrator ports when terminal locations warrant it practical as is done on the GE 34 
network [11].

The least attractive possibility for a network interface is the use of the host computer itself. This situation occurs when no special terminal interface has been developed for the network so that terminals can access the network only by connecting to their host computers as is presently the case with MERIT [24] and TSS [25]. This implies that to communicate with a remote system, processing must be done by both the remote system and the users host system, driving up overhead and costs.

Message switching computers are also relevant to the discussion of communications hardware. In order for a user to establish a connection with a particular computer, he can dial a unique number to make that connection, the number being different for each computer to which he wishes to connect (the number can also be given to a computer which will dial it by using an automatic calling unit). If a user, however, is to dial only one number, or be connected to one leased line regardless of which computer or computers with which he wishes to communicate, then a switching computer must be employed in the network architecture. The switching computer performs the store-and-forward function, that is, a message is received enroute, stored only until the proper outgoing line is available, and then retransmitted. In the ARPANET, for instance, the design end-to-end delay through several store-and-forward nodes is less than 0.5 seconds [26]. 
Each message contains an identification of the sending device and an address for the receiving process allowing the switching computer to route messages from any source to any destination. The switching function can also be performed by mapping the input ports of the switching computer to the appropriate output ports [20]. Besides the inherent flexibility in software switching, the store-and-forward technology allows for optimal line utilization, since interprocess messages can be mixed with interprocess messages involving different host computers and terminal devices on the network. Also, the switching computer can perform other functions such as concentrating terminals and interfacing to service computers.

\subsection{Communications Software}

Communications software can best be discussed in relation to the control it exhibits over a network. The ARPANET uses a we11-structured layered approach [27] and the terminology describing this structure is useful in discussing network communications software. At the lowest level is the synchronous communications protocol governing the exchange of information between switching computers [19]. Next, the so-called first level protocols control the exchange of information between a host computer and its interface. Using these two protocols in its implementation is the level two protocol controlling the exchange of information between host computers [28]. Finally, there are the level three protocols which control the information exchange between actual processes where a process 
may be a computer program or a user terminal [27]. At this level are the protocols which are very relevant to the user in relation to the utility of the network. In the case of ARPANET third level protocols exist to allow for initial connection to a remote process, to control communication between a terminal process and a computer process, to transfer data and files between processes, and to define remote job entry communications.

For a network to truly be a utility, there should exist in the hierarchy, a network control language which is concerned with human considerations related to the use of the diversity of systems that can exist on a network. In the network control language there should be a set of standard commands to perform common simple and intermediate system control functions regardless of the system being used on the network. For example there is a set of simple functions such as logging in, listing files and directories, etc., which should certainly be standardized in addition to some of the intermediate functions such as running common compilers and executing jobs.

The control functions from the lowest level on up can be distributed to various network components. It is useful to consider different possibilities to aid in understanding some of the concepts applied and the relationship of alternatives to networking considerations. If switching computers are involved some network component must be responsible for establish- 
ing an initial connection between two processes desiring to communicate (a customer terminal and a host computer program for example). This type of control can be centralized in the network (even though topologically the network is distributed), such that every process desiring to communicate with another process on the network will do so by initially connecting to the same computer which will establish the desired connection [20]. Since a single computer is establishing all initial connections it may be more convenient to approach standardization in the connection mechanism relieving the user of the burden of learning a variety of protocols for the different systems on a distributed network. There is, however, enormous dependency on that single computer, and it is essential that backup systems be incorporated into the architecture. Another possiblity is to allow the interfaces to the network of the involved processes to be responsible for establishing the initial connection, thus distributing that function to all nodes on the network [23]. This removes the dependencies of those connections from any one computer implying that failures will cause only local disturbances.

The control of traffic flow through the network is another software function. The traffic flow between the various communications components will be typically controlled by ordinary line control procedures [29]. The flow control between processes can involve special software in the computers on which those processes are running. This is the 
situation with the ARPANET where interprocess flow control is host resident [28]. An alternative is to use the regular software controller of a particular computer to control interprocess communication. This can be done if al1 of the computers and systems are similar [25] or if a front-end device performs a mapping function between the network and the software controller [24]. Another alternative is to have a central computer be responsible for flow control accepting inputs from al1 processes in remote computers desiring to communicate [21]. The separation of communicating processes and the computer or computers which regulates the traffic flow between them can lead to the capability of sending information to a process on a computer which is not at the time available.

When a message can transverse more than one path in a network from a source to a destination, then the communication software must perform a routing function. If the network is circuit switched, then a physical connection is established at each switching point, and remains so established for the duration of the connection [25]. The software involvement is minimal, in this case, and can, for example control an automatic calling unit. If a logical or virtual circuit is established, then communications software residing in some network component or components must select the optimal path for the establishment of that circuit [20]. This can be done by comparing the conditions of the possible circuits to select that with some combination of least traffic and least errors. 
In a message switching network, each individual message, is routed dynamically, or as in the case of ARPANET, messages are divided into packets, each of which is dynamically routed. Considerable attention has been given to the development of routing strategies for a packet switched network such that circuits are efficiently utilized and high throughput can be obtained $[19,30]$.

Another facet of network control is the measurement of network performance. It is possible to generate statistics to monitor the internal performance of the communications network, for example, queue lengths, message sizes, routing, etc., to aid in tuning the system and providing fault detection and correction $[31,32]$. Another set can be recorded to provide more of an external measurement on network usage [4]. Recordings of traffic flow between nodes can be analyzed to determine proper distribution of facilities including placement and number of service computers, switching computers, and device interfaces, and bandwidth and topology of communication circuits. In addition usage statistics can be kept of the different processes available at the service sites and of the generic types of services available such as timeshared, batch, or graphics. Both the internal and external measurements will aid in determining the effectiveness of the computer network and the appropriate distribution of services. 


\subsection{Configuration}

The preceding discussions have described network parameters which identify the network technology at a local level in terms of its components. To get a more global representation of a network technology, it is helpful to examine networks in terms of configuration and configuration alternatives. Alternatives will be considered relating to network topology, composition, size, and distribution.

Network topology can be centralized or distributed. Centralized networks, also called star networks, are those in which all nodes connect to a single node. This is a description of topology, only, and implies nothing about location of computing power, network control, or user distribution. The alternative topology is distributed where, in the limit, each node is connected to every other node, although the terminology is commonly applied to topologies approaching this full connectivity. There exists a third alternative which more accurately describes a certain condition of a distributed network. This is the ring configuration commonly used in describing the Distributed Computer System [33]. Observing the topology a ring can be seen connecting the nodes of the network. In the case of TYMNET a number of such rings are then themselves interconnected forming a multiring structure [20]. This is a special case of a distributed network.

For a network of distributed computers, a distributed topology would seem more practical for connecting existing computer 
centers, in addition to the reliability factors that favor a distributed network. If it were the intention in building a network of constructing one large and reliable computing facility to which all customers could connect, then a centralized (or some modification of a centralized) topology might be in order [34]. If the intent is to connect to existing distributed centers, and not radically disturb the balance of computing power, a distributing topology is implied. There exists a potential arrangement using advanced communications technology where a network can be a centralized network while the computing power remains distributed. Consider using satellite communications to replace existing earth-bound longline circuits. Transmission from any user (human or computer) to any process can travel to a satellite and, using the satellite's broadcasting ability, to the target [13]. This topologically, is a centralized network that allows for distributed computing power, and worth mentioning because a potentially powerful transmission medium is considered. To avoid ambiguities it is probably helpful to qualify statements of centralization or distribution with references to topology, computing power, switching, and network control. In the satellite example distributed computing power with central switching is the desired description.

As the terms composition, size, and distribution are fair1y descriptive, 1ittle development is necessary other than the definition of alternatives. Network composition can be homo- 
geneous or heterogeneous referring to the host computers on the network. If the network is homogeneous then all host computers are the same and if heterogeneous, all are not the same. The term heterogeneous is sometimes applied to networks having computers which are different models from the same manufacturer [21]. For this report, the most meritorious are those that are capable of connecting to the majority of existing large scale computing systems and not just different models of a computer. One of the details included in the survey in chapter 4 of existing networks will be a 1 ist of the actual computers connected.

The size of the network in terms of the number of nodes and distribution or actual location of those nodes is also important. For purposes of this report, it should be evident that a network can support on the order of thirty (30) to fifty (50) nodes with a national, and possibly, international distribution.

A number of technical factors have been identified to describe a network communications technology and characteristics of configuration have been discussed, and at the same time, a working terminology has evolved. Whenever possible, technological factors were considered independent of the other factors comprising a network technology, and evaluated using generalities as to their effective application in the Networking for Science Program. It is now possible to investigate existing networks and discuss the technology applied using the 
aforementioned terminology subsequent to the comparison of those varied approaches and the technology or technologies that they represent. 
4. EXISTING NETWORKS

It was not considered practical to attempt to review every existing network. The networks chosen are considered a fair representation of the various current approaches to network technology for networks of computers having potentially national scope. It is important to distinguish between a technology, which is the primary concern of this paper, and implementations based on that technology. This distinction is necessary because networks not included in this report may be reasonable alternative implementations employing the technologies represented by those chosen.

In this section selected networks are classified in terms of the technology or technologies that they represent. For each network, following a brief overview accompanied by graphical representations of the network, the communications enployed will be identified in relation to communications circuits, communications hardware and communications software using terminology developed in the previous section. Following this is a description of the network configuration, again relying on the aforementioned terminology. Then, if a network possesses any salient features related to the technology employed and in the author's opinion, particularly noteworthy they will be itemized. Finally, the network technology will be related to the previously discussed desirable support capabilities for a computer network. 


\subsection{TYMNET}

TYMNET is a distributed network of heterogeneous computers (see Figure 1). It exists primarily to connect the users of the time-sharing service of Tymshare, Inc., to the computers of Tymshare. However, because of the wide distribution of communications facilities and the flexibility in host and terminal interfaces the network lends itself nicely to the connections of non-Tymshare host systems and their users nationally distributed. This is different from the kind of service that can presently be obtained from common carriers in that value is added to the carrier circuits because of the front-end, store-and-forward, error detection and retransmission, and concentration processing capabilities of the mini-computers which are an integral part of the network.

\section{Overview}

The network consists of approximately ninety (90) minicomputers, Varian 620's, interconnected by common carrier voice grade facilities. The Varian 620, when employed in TYMNET, is called a Tymsat and serves in two separate capacities, as a base Tymsat and as a remote Tymsat. The base Tymsat is responsible for acting both as a message switching computer in the communications network and as an interface to the network for host or service computers. When the host computer is one of those generally supported by Tymshare, such as an XDS 940 , 
DEC PDP-10, or IBM 370, the Tymsat has been programmed as a communications controller replacing the standard components for that function. Computers of a kind not employed by Tymshare have thus far been interfaced to the net through the Tymsat where the Tymsat sends and receives input and output appearing as a rotary of asynchronous modems to the standard communications controller for that machine, e.g., an IBM 270X or 3705 for an IBM 360 or 370 . Present1y under development is a Tymsat to replace the IBM communications controller. This will eliminate the need for the IBM $270 \mathrm{X}$ or $370 x$ and allow for greater flexibility in terminal support. The base Tymsats are each connected to a host computer in either a one-to-one or one-to-two fashion (connecting approximately forty host computers throughout the country) and to one another either directly or through an intermediate base Tymsat in a multiple ring or distributed manner. The circuits used are from 2400 to 9600 baud synchronous, full-duplex, private leased lines.

\section{Terminal Interface}

The remote Tymsats act as store-and-forward computers and as concentrators for user terminals. Each is capable of supporting up to thirty-one (31) asynchronous full-duplex modems allowing for terminal speeds in the 110 to 300 baud range. In addition, some remote Tymsats with added hardware and software can support hardwired terminals in the 600 to 1200 baud range. The remote Tymsat can identify a terminal 
by the first character typed as to its baud rate and carriage return delay time. It is possible to allow a terminal to connect with two different baud rates, one for input and one output. In addition, conversion to and from ASCII with the accompanying overhead is provided for EBCDIC or Correspondence coded terminals. The remote Tymsat also performs echoing when a user terminal is operating in echo-plex mode. The remote Tymsats are connected to the base Tymsats, and thus the host computers, in what is best described as a multiple ring configuration. A circuit passes through a number of remote Tymsats and one base Tymsat using store-and-forward techniques to exchange information between any remote Tymsat and any base Tymsat. The high speed links to the remote Tymsats (versus the low speed links which connect the terminals) are again 2400 to 9600 baud synchronous, full-duplex, private leased lines. Data is blocked and buffered and the usual error checking and retransmission, as necessary, is incorporated allowing for virtually error-free transmission between Tymsats. Error detection between terminal and Tymsat is accomplished through character echoing.

\section{Character Transmission}

When transmitting interactive traffic in a network there are two related problems for which TYMNET has implemented an interesting solution. The first is concerned with transmitting characters in echo-plex mode, which is almost always the situation when interacting with full duplex systems. The 
problem is to echo a character without any uncomfortable delays to the user. An uncomfortable delay would result, for example, if a user typed two characters in succession before the first was echoed. To meet this "instantaneous" echo requirement it is necessary to transfer a character through the network to the service computer, assigning the character and its echo a high priority. of course, transmitting a single character is quite costly in terms of overhead since each unit of transmission must contain the appropriate header and error checking information. This is to identify the data as to source and destination and to insure its integrity. The TYMNET implementation provides a solution by having the remote Tymsat (that to which the terminal is connected) perform all echoing requiring immediate response (i.e., type ahead does not require immediate response). The second problem is that of reducing the "overhead to data" ratio in message transmission. Remote echoing helps by making it possible to buffer, prior to transmission through the network, an entire line of input from a user; because it is the line and not each character in the 1 ine that requires a response. The TYMNET approach to the blocking of data goes further to ameliorate the overhead problem by collecting, at each Tymsat, the input from multiple sources destined for the same output port, to form a single block. The maximum block size is 66 characters and the attempt is to approach that maximum prior 
to transmission thus increasing efficiency in terms of the message overhead to data ratio. Of course there is computational overhead at each node (Tymsat) since sending nodes must assemble the characters of a block and receiving nodes must disassemble them.

\section{Virtual Circuits}

Blocks are transmitted through the network over ful1duplex virtual circuits. These circuits are established at log-in time and exist for the duration of the interaction between terminal and processor. The circuits are not physical but exist as table definitions in the software of involved Tymsats associating an input channel with the appropriate output channel at each switching point (Tymsat). A circuit is established by the network supervisor (computer program) in active mode (Sam). TYMNET provides multiple paths for circuits in the event of line failure. When a user connects to TYMNET he is originally communicating with the Sam which after the appropriate exchange of information will establish a circuit from that user's terminal to the proper service computer by selecting the appropriate Tymsats to complete the virtual circuit. The Sam is so named because it is a function which can be overtaken by one of three backup supervisors in the event of failure. A backup supervisor will become active by detecting a failure, polling the Tymsats to get network status information, and assuming the active role. This is performed transparent to users 
other than those who may have been connected to the processor running the Sam that failed. It should be noted that in the event that a processor fails, its base Tymsat can still function in the role as a network store-and-forward computer. Since virtual circuits are established and fixed for the duration of processor interactions, recovery from a physical line failure is not as clean. When a line fails, users communicating through virtual circuits using that line in their definition must reconnect.

At this time the TYMNET computer network can have 900 individual users simultaneously connected to the network. The communications network exhibits a high degree of reliability with a stated average 1.4 failures per node per year. Presently, most usages are through interactive terminals because the TYMNET does not support remote job entry. However, high-speed printers exist in many field offices to which large volume printing may be routed. 


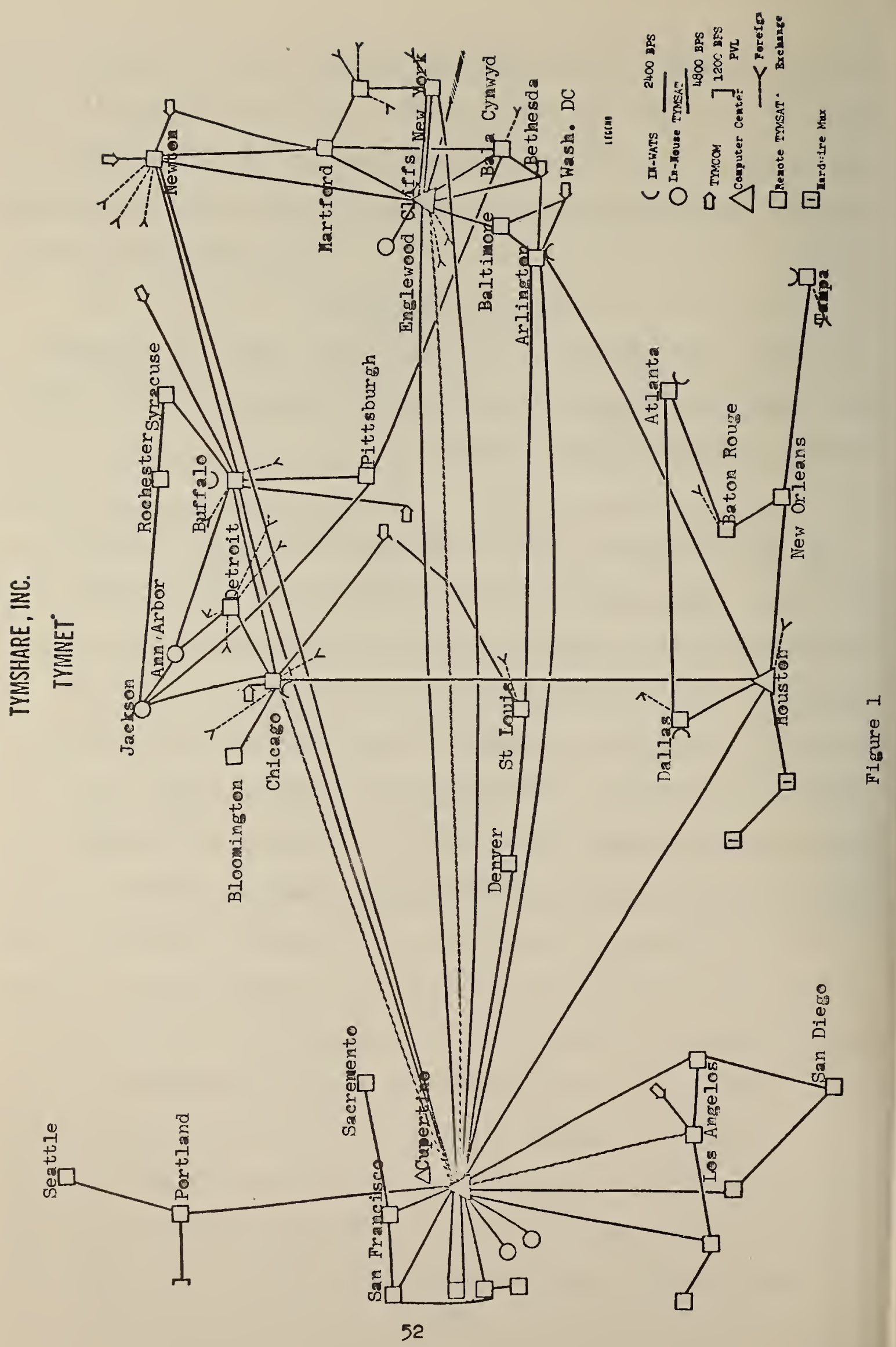




\section{Communications}

Communications Circuits

Internode - circuits are voice grade, leased, synchronous operating at $2400,4800,7200$, or 9600 baud.

Terminal to network - circuits are full duplex dial-up or hardwired operating at 110 to 300 baud (dial-up), 600-1200 baud (dial-up in certain cities), and 600-1200 baud (hardwired).

Interprocess - virtual circuit established at log-in time by the network supervisor in tables in the store-and-forward nodes.

Communications Hardware

Single host interface - a Varian 620 ( $8 \mathrm{~K}$ of 16 bit words, 1.8 usec cycle time) called a base Tymsat, also acting as a store-and-forward node.

Dual host interface - a Varian 620 L-100 (12K of 16 bit words, $1.0 \mu$ sec cycle time).

Terminal interface - a Varian 620 called a remote Tymsat also acting as a store-and-forward node. Each remote Tymsat supports up to 31 asynchronous, full-duplex terminals and performs speed recognition, local editing, and character conversion. 
Communications Software

Initial connection - all users initially connect to the same processor, the supervisor in active mode, (Sam), which will establish the optimum communications circuit between the user and the appropriate processor on the network. Alternate routing occurs during leased line outages. The Sam also acts as a service computer on the network.

Protocols - computer processes communicate by looking like terminals to one another. 


\section{Configuration}

Topology

Distributed - service computers, store-and-forward nodes, and user terminals are well distributed and interconnected in a multiple ring configuration.

Actlal distribution - international connecting 53 cities throughout the nation in addition to a link to Paris, France.

Composition

Heterogeneous - XDS 940

XDS Sigma 7

DEC PDP 10

DEC PDP 9

DEC PDP 15

IBM $370 / 155$

I BM $370 / 158$

IBM $360 / 40$

IBM $360 / 50$

IBM $360 / 65$

IBM $360 / 67$

Burroughs B6700

Honeywe11 G275

Univac 1108

CDC 6400 
Size - approximately 40 host computers, over 90 communications nodes serving 900 individual users simultaneously. 


\section{Salient Features}

TYMNET is reliable with an average number of failures of 1.4 per year for a Tymsat and an error rate of less than one bit in $4 \times 10^{9}$ bits transmitted.

The communications facility is efficiently utilized supporting approximately 46 users operating at speeds of 110 to 300 baud on a 2400 baud circuit.

Terminal recognition is automatic with baud rate, character rate, and carriage return delay time detected and input and output speeds may be set independently.

The network supervisor in active mode which controls all connections can be transferred in the event of failure to another processor without effecting users. It maintains the current status of all network activities.

TYMSHARE will lease the communications service (TYMNET) exclusive of its host systems, allowing a customer to connect his own computer to the network to serve his own users. This service is charged for on the basis of value added to common carrier facilities and operates under FCC Tariff Number 260, Shared Facilities. The service is presently used by many organizations including the National Library of Medicine in Washington, D. C. 


\section{Terminal Support Capabilities}

Interactive

Code - network code is ASCII although terminals may operate in ASCII, EBCDIC, or Correspondence code. Speed - 110 to 300 baud dial-up and 600 to 1200 baud hardwired (requiring on-site Tymsat).

Mode - full-duplex.

Remote Job Entry

RJE support does not exist but is planned for future implementation. A synchronous protocol may be developed in a Tymsat-like device to support RJE devices of the IBM 2780 variety.

Minicomputer-based Systems

No minicomputers are on the network other than as an integral part of the communications net (Tymsat). There is no support for synchronous communications at the user level and the bandwidth is presently limited to 9600 band on the high speed circuits.

Computer-to-Computer

One computer can connect as a terminal to another and vice versa allowing for interprocess communication. Files can be transferred from one processor to another in this manner. 


\subsection{CYBERNET}

CYBERNET is a commercial network of large to super scale Contro1 Data Corporation (CDC) computers and it operates as a division of $\mathrm{CDC}$. These computers have been connected to a communications network (see Figure 2) to form a national computer network satisfying a number of important objectives. Reliability is increased by allowing a user to access an alternative computer in the event of a failure of his loca 1 computer. Workloads can be leveled among the participating systems by routing jobs from loaded systems to unloaded ones. Computers can be better utilized by specializing in applications to which they are best suited and the network allows for the routing of jobs requiring those applications to the proper computers. Large data bases can be kept at selected sites and need not be copied at each site to be accessible since the network will provide access as required. Also access to the super computer CDC 7600, when connected, will be more generally available with the national CYBERNET network. This presentaion is limited to the U.S. but CDC is evolving similar networks using the same technology in Europe and Australia, and on a smaller scale, in other countries

\section{Overview}

The technology employed by CYBERNET is difficult to characterize. It is heterogeneous in that it interconnects different models of Control Data computers including the 
$\operatorname{CDC} 6600, \operatorname{CDC} 3300$, and $\operatorname{CDC} 6400$, and plans exist to connect a CDC 7600. The communications links interconnecting computers are a combination of voice grade and wideband, and even include a satellite link. The switching mechanism employed is mostly line switching but a special use of message switching exists. The topology, control, switching, and computing facilities are all distributed. CYBERNET appears to be an add-on network where new technologies are incorporated as needed, void of commitments to particular hardware, software, or communications facilities.

\section{Centroids}

The bulk of the computing power for the network is performed by the centroids. The centroids are all currently CDC 6600's operating under SCOPE operating system, although because of the SBC acquisition, plans call for connecting IBM 370's. Access to the centroids is primarily via wideband batch terminals (see "Terminal Interface"). The centroids are interconnected with full duplex 40,800 baud wideband communication links. The switches employed are multiway wideband switches. These switches connect to each other in a distributed fashion, to the centroids, one per switch, to the nodes, one or more per switch, and to the wideband batch terminals. The CYBERNET concept of nodes will be discussed later. The switches allow the terminals to access the facilities of the network providing flexibility in that the terminal can connect to any centroid, and provid- 
ing down time back up and load leveling.

It should be pointed out that load leveling is not automatic, but involves a human decision to use, by dialing, one facility rather than another. Also down time back up for any job is entirely dependent upon that job not using permanent disk files, since the file systems are host dependent.

\section{Nodes}

The term node as used in CYBERNET refers to computers which act as processors and store-and-forward computers. The nodes are actually CDC 6400's using the KRONOS timesharing operating system. Their main purpose is to provide time-sharing service to low-speed interactive terminal users. It is, however, possible to access the batch computers CDC 6600's - from a low-speed terminal indirectly through the CDC 6400's. Jobs can be submitted via terminal to the CDC 6400 KRONOS time-sharing system destined for the CDC 6600 SCOPE batch system and KRONOS will forward the job to SCOPE using a wideband switch. One paper presents plans for the nodes to have a number of important capabilities for load balancing and resource sharing [10].

\section{Termina 1 Interface}

Terminal support on CYBERNET includes support for interactive or conversational terminals, remote peripheral processors, satellite computers, and computers with terminal facilities. The interactive terminals are those of the 110 
to 300 baud range and using ASCII. The remote peripheral processors are conventional computer peripherals driven at a remote site. The typical configuration is a card reader, a line printer, and a modem controller. Supported are low speed (2000-2400 baud), medium speed (2400-4800 baud), and high speed (40,800 baud) devices using 300 card per minute (CPM) and line per minute (LPM) readers and printers, 600 CPM and LPM readers and printers (planned), and $1000 \mathrm{CPM}$ and LPM readers and printers respectively. The CDC 200 User Terminal is the standard CYBERNET device for low and medium speed remote peripheral processors. It is a hardwired rather than programmable peripheral device. The satellite computers are programmable peripheral processors and appear to the network as the hardwired remote peripheral processor. They are distinguished by their ability to do off-net processing and, when on-net, can support the higher speed readers and printers. The satellite computers presently used number over 25 including: Honeywe11 200 series, Univac 9200's, and IBM 1130's. The last class of terminals are computers with terminal facilities which are computers which have been programmed to behave as terminals to the network. This is the manner in which the larger scale IBM $360^{\prime} \mathrm{s}$ access the network. At this time nearly 800 remote peripheral processors, satellite computers and computers access CYBERNET. In addition the CDC 6400 KRONOS system and the CDC 6600 SCOPE are programmed to appear as terminals 
to the net while simultaneously serving terminals connected to them.

The look alike technique is important for computer-tocomputer communications on CYBERNET. The standard remote peripheral processor for CYBERNET is the CDC 200 'User Terminal, but other devices have been programmed to $100 \mathrm{k}$ to the network like the CDC 200 including: CDC 8090 , CDC 160A, IBM 1130, UNIVAC 9200, UCC's COPE, Honeywell 200, and IBM $360 / 30$ and larger IBM $360^{\prime} \mathrm{s}$. The nodal computers can look like wide band terminals to the centroids and to each other. This is an uncomplicated method of performing computer-tocomputer communication and renders it less difficult for incorporating new systems into the network. 


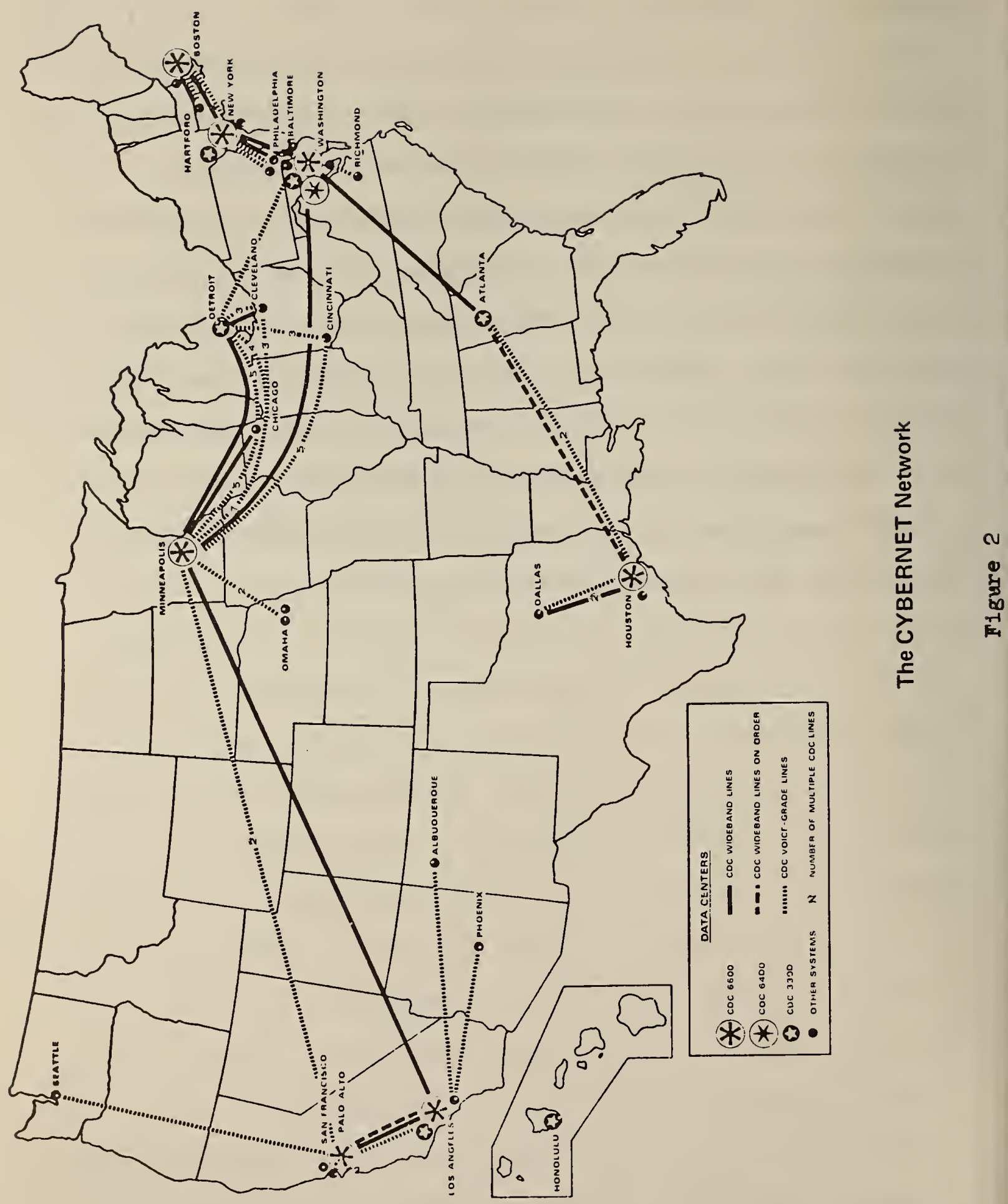


Communications Circuits

Internode - full-duplex 40,800 baud wideband facilities between wideband switches. A plan calls for switching nodes being developed which will initially be connected by 2400 and 4800 baud 1ines [10].

Terminal to network - voice grade operating a 110 to 4800 bits per second and wideband facilities at 40800 bits per second to connect terminals which include interactive terminals, remote peripheral processors, and satellite computers.

Interprocess - al1 interprocess communication, node to centroid for example, is via a look alike technique where one process appears as a standard network terminal to another. These communications are established by direct connection or connection through a wideband switch.

Communications Hardware

Host interface - multiway wideband switch can interface host or hosts to the network.

Terminal interface - terminals connect (multiplexed or individua11y) through the host computers, the nodes, or the wideband switches. Ter- 
minals can be computers.

Communications Software

Initial connection - user selected through appropriate

settings on wideband switch. Also done automatically by nodal computers.

Protocols - computers communicate through look-alike techniques appearing as network standard terminals or peripherals to the network. 


\section{Configuration}

Topology

Distributed - nodes, centroids, and user terminals are distributed.

Actual distribution - computer centers exist in at least 12 cities around the country and 1 inks exist to over 45 cities including Honolulu and Anchorage, Alaska. KRONOS systems are in Washington (3), and Los Angeles and over 30 metropolitan areas are covered with local phone service.

Composition

Heterogeneous - CDC 6600

CDC 6400

CDC 3300

Peripherals - IBM 360

CDC 3150

CDC 8090

IBM 1130

UNIVAC 9200

Honeywe11 200

Size - at least 9 CDC 6600 SCOPE batch computers and 4 CDC 6400 KRONOS time-sharing computers with a large number of users (approximately 600 simultaneous users on KRONOS and 250 RJE stations on SCOPE). 
Many companies add value and resell at a higher price via terminal the facilities of CYBERNET and do therefore not have to have their own computers to be in that business. Bulk discounts apply.

Several packages are offered through CYBERNET by independent companies on a royalty basis including a dynamic structure package, a time series analysis package, a 1970 Census Tape processing package, a data management system, and a crystallography package.

Super computer processing power is made available on a nationally accessible basis to the paying customers of CYBERNET. 


\section{Terminal Support Capabilities}

Interactive

The code is ASCII, speeds are in the 110-300 baud range although nothing in the design precludes higher speeds, and the mode is half-duplex.

Remote Job Entry

Support is extensive centered around the CDC 200 User Terminal which is a non-programmable device supporting a card reader, a 1 ine printer, and a modem controller. Other devices can be programmed to look like this device.

Card readers and 1 ine printers operate in the speed range of 300-1000 plus cards per minute and 1 ines per minute.

Minicomputer-based Systems

Minicomputers have been programmed to look like either remote peripheral processors or as so many terminals to the network.

Computer-to-Computer

Computer communicate by look alike techniques to the network. 
4.3 GE Information Services

The General Electric network is a commercial network implemented to make available the computing services of General Electric Information Services. The network encompasses over 300 cities in North America, Japan, and Europe, making it international in scope. The communications network allows a user to make a local phone call to connect to the main computing facilities in Cleveland, Ohio, providing services ranging from light computation to remote batch processing. The network has the interesting capability of connecting customer in-house computers to GE network service computers for the purpose of exchanging files. This will be discussed later.

\section{Overview}

The GE network is a centralized, hierarchical network. Topologically, all communications paths lead to the central facility. The central facility itself is composed of a number of processors with varying capabilities, switching computers which allow flexibility in the choice of userprocessor connections, and central concentrators which establish initial connections and act as interfaces between the processors and the network. There are presently two switching computers and each central concentrator is connected to both of them. They are able to switch messages between central concentrators. Each central concentrator acts as a network interface for from one(1) to six (6) 
processors. Moving out from the central facility, each central concentrator can connect to a maximum of eight (8) remote concentrators. At the present, more than fifty (50) remote concentrators are in service. The remote concentrators typically have forty-eight (48) ports connected to the public switched network using local loops, foreign exchange, and frequency division multiplexers. The FDM multiplexers can multiplex up to nine ports on a voice grade circuit. Finally, there is the user at his terminal who can dial a local number and through the hierarchy of communication, establish a connection with the desired processor.

\section{Remote Concentrators}

The remote concentrators are Honeywe11 416 or GE Diginet 1600 minicomputers. They act as network interfaces for terminals of speeds up to 300 baud. They are responsible for recognizing terminal speed, code, and other characteristics. A11 non-standard codes are converted to ASCII before transmission onto the network. There is no echo'ing (characters are printed by the terminal when a key is depressed) and transmission is line at a time, that is, characters are buffered until a 1 ine is completed before transmission on the network. The remote concentrator can also perform line and character deletion editing. In addition, it is able to recognize whether a terminal is entering a command or data (as in file building) and only interrupt the central system 
when a command has been entered. Each remote concentrator is connected to a central concentrator by a pair of 4800 baud voice grade lines. There also are being developed remote high speed concentrators using Diginet 1600's [11]. These will support synchronous terminals up to 4800 baud. Included in the set of synchronous terminals are computers, and in particular, an os teleprocessing package has been developed to permit IBM 360 or IBM 370 computers to interface through the high speed concentrator. The high speed concentrators will initially connect to the central concentrators over dual 1 ines at 9600 baud. At this time remote job entry devices and IBM 360 or 370 computers connect to the central facility via 2000 or 4800 baud dialup circuits. Central Facilities

The central concentrators are Gepac-4020's. They storeand-forward data to and from terminals in their role as interfaces between the host and the network and also between remote concentrators and the network. Host or central computers are connected in a variety of ways depending on the host involved including direct memory to memory interfacing and remote communcations channels using synchronous communications protocols. The central concentrator determines to which system the user is to be connected at $10 \mathrm{~g}-$ on time. If it is a system that is not connected directly to the concentrator, then the concentrator will send all 
messages from the user to a switching computer which will examine the destination address for each message and route it accordingly. The circuits are two-line links in the speed range of 4800 to 14400 baud. The switching centers which provide the connections between concentrators are Gepac4020's or Diginet $1600^{\prime} \mathrm{s}$. There presently exist two switches which operate independent1y. The host or central processors themselves can be either G635's, G605's, G265's, or HISI $6000^{\prime} \mathrm{s}$.

\section{Computing Services}

The network and its central system offer different kinds of computer services depending on the host computer being used. The Mark I time-shared system offers BASIC, ALGOL, and a simplified version of FORTRAN. The Mark II timeshared service is an outgrowth of Mark I and offers extended capabilities including geographically distributed transaction oriented processing, and it runs on the Honeywel1 G635. The most interesting service is the Mark III which combines the Mark II time-sharing system and the GECOS III remote-batch system running on the larger Honeywell 6080. All on-line activities are handled by the Mark II (called Mark III foreground) which is optimized for that kind of work. Large batch tasks are done by GECOS (called Mark III background) likewise optimized for batch. A high-bandwidth interface allows background work to be submitted by Mark II across the interface to GECOS. Therefore, batch jobs originating from 
low or high speed terminals connected to Mark II, can be run on the background GECOS and the output sent back to a file on Mark II where a user can print it on a high speed printer or scan it with his terminal. The high bandwidth communication channel between Mark II and GECOS is actually a shared disk device. This is not to imply that the two have a common file system, on the contrary, the file systems are independent and distinct.

\section{Interprocessing}

GE's facility known as Interprocessing is an exciting concept. A customer is permitted to use his in-house computer for production-type processing, then transfer his files through the GE network to the central computers, to make them available on-line throughout the international network for customer interactive query and update. Periodical1y, the updated files are transferred back to the customer's in-house computer. Pontiac Division of General Motors uses the Interprocessing capability of the network maintaining order and delivery information for its thousands of automobile dealers [35].

For the future, there are indications that GE will expand the Mark III foreground-background concept to include other manufacturer's equipment for running the background jobs, including IBM systems and possibly the Control Data 7600 [34]. Also considered is the possiblity of supporting remotely 
located background systems such that a customer owned and operated computer could be connected to the network as a background system. This would allow a customer to couple his machine and applications to the massive on-line capability of the GE network. 


\section{Communications}

Communications Circuits

Remote to central concentrator - dual 4800 baud voice grade.

High speed remote to central concentrator - dual 9600 voice grade.

Central concentrator to switching center - dual links in

the speed range of 4800-14400 baud.

Terminal to network - maximum for asynchronous terminals

is the 300 baud and 4800 baud for synchronous.

Low speed terminals connect to frequency

division multiplexors which connect to the

remote concentrators.

Interprocess - user terminals are connected through their

remote and central concentrators to the pro-

cessor or processors connected to that

central concentrator or, using message

switching, are connected to processors on

other central concentrator's ( concentrators

to which their remote concentrators are not

directly connected).

Communications Hardware

Host interface - hosts interface to the network through

the central concentrators which are GEPAC4020's. More than one processor (six maximum) can be connected to a central concen- 
trator.

Terminal interface - asynchronous terminals, after multiplexing, interface to the network through the remote concentrators which are Honeywe11 416's or GE Diginet 1600's. Synchronous terminals will in the future interface through high-speed concentrators using Diginet 1600 's. At this time synchronous terminals must dial the central concentrator facilities.

Communications Software

Connection - central concentrators connect user terminals to the appropriate processor using information in the user number.

Traffic flow regulation - distributed between remote and central concentrators.

Protocols - computers can connect to the network using BTAM (Basic Terminal Access Method) operating under OS. This facility is limited to IBM $360^{\prime} \mathrm{s}$ and IBM $370^{\prime} \mathrm{s}$. 
Topology

Centralized - all communications feed into a central system in Cleveland. Within that central system the compute power is distributed among a number of processors.

Actual distribution - the network extends via local telephone to over 250 cities in the U.S., nine cities in Canada, Mexico City, San Juan, London, Birminghan, Manchester, Brussels, and Amsterdam. •

Composition

Heterogeneous - GE 635

GE 605

GE 265

HISI 6000

(plans call for background systems employing IBM and CDC machines [34]).

Size - Approximately 50 remote concentrators in 13 U.S. and European cities. Each concentrator can have 48 user ports. Maximum capacity is on the order of 2000 simultaneous users. The total customer base is 3000 firms with over 10,000 individual users [7]. 


\section{Salient Features}

A service called Interprocessing is offered in which

files from a customers own computer may be transferred through the network to the GE system computers for on-1ine access by the customers terminals, possibly, distributed throughout the country. Updated files can be periodically sent back to the customers computer.

In addition to the time sharing services, background systems, interfaced to the network through the time sharing systems, run batch jobs submitted via terminal or remote job entry stations.

Plans call for the introduction of IBM and possibly CDC equipment into the network for running background jobs lessening incompatibility problems with customers facilities. 


\section{Terminal Support Capabilities}

Interactive

Code - ASCII, EBCD, Correspondence.

Speed - 10, 14.8, 15, and 30 characters per second. Mode - half-duplex.

Remote Job Entry

Synchronous support is provided for the IBM 2780 binary synchronous line discipline and compatible devices. The high speed code is EBCDIC. The connection is via 2000 bit per second dialup to the central facility although high speed remote concentrators are being developed. Minicomputer-based Systems

Synchronous interfaces and sufficient bandwidth exists to support these systems although no such applications presently exist.

Computer-to-Computer

With the appropriate communications adapter (IBM 2701, 2703, or a 3705 emulating a 2701/2703) and a GE-provided software package, certain computers can connect to the high speed service as does a remote job entry device. These are all IBM $370^{\prime}$ 's and IBM 360's models 22 and up using IBM OS-BTAM. The interconnection is used mainly for large file transfers. 


\section{4 MERIT}

MERIT is a state network developed with funds provided by the National Science Foundation, the State of Michigan, and the three participating universities: Michigan State University, University of Michigan, and Wayne State University. It interconnects the computing facilities at these three universities, IBM 360/67's at two nodes and a CDC 6500 at the third (see Figure 3 ), and provides a working model to study the problems associated with the use of remote computing facilities, that is, the use of computing facilities at a university different from the university of the user. The several problems being analyzed, in addition to the technical development of the network, include the following: the provision of a mechanism for exhanging computer dollars among the universities; the inclusion of the smaller state universities into the network and finding a suitable mechanism for payment of the communication facilities; and the problem of informing potential users of services that are available on the network and the detailed procedures of using the network resources [36].

\section{Communications Computer}

The technology used in this network goes beyond the much simpler directly connected host-to-host network by employing communications switching computers which act as interfaces between the host computers and the network, and also as store-and-forward nodes. The Communications Computer 
is a Digital Equipment Corporation PDP-11/20 with $16 \mathrm{~K}$ of $16-$ bit memory. The primary purposes of the Communications Computers are the following: to multiplex logical connections over physical communications links communicating with other nodes using a binary synchronous communications protocol, to receive messages from their respective host computers for transmission on the network, and to receive messages from other nodes for delivery to their hosts or for forwarding to another host.

The interface between the Communications Computer and the host computer is a special hardware device. It allows for the transmission of software determined length data records to and from the memory of the Communications Computer (CC) performing whatever memory alignment operations are required for word lengths which differ from that of the CC. It also provides a multi-address capability which allows the host to treat the CC as many identical devices. This simplifies the task of programming the host computers since the CC does all the multiplexing functions.

\section{Network Control}

To interface a host to the network requires no changes in the operating system of that host computer. Instead the network features are extensions to the operating systems, requiring, only that device support for connections, which appear similar to other peripheral devices, be added to the system. Therefore, when a connection is established be- 
tween processes in independent host computers, the connection behaves as an input/output device in each host. An initial connection is established to prepare the "connection device" for record traffic by a single user request. The CC propagates this connection request through the network to the remote process and then the connection is completed with the allocation of a "connection device" to each process. Traffic is exchanged through the read/write mechanisms of the host computers under the control of a network master/slave mechanism to regulate traffic flow. The master, the remote end of the connection, can always initiate the sending of a record to the slave. The slave is restricted to send one record on each go-ahead from the master. The record traffic regulation mechanism, as can be noted from the preceding, is resident in the host computers.

\section{Circuits}

The original communications circuits interconnecting the CC's were 2000 baud dial-up facilities. Each CC was interfaced to a maximum of eight (8) direct dial telephone lines. To establish full duplex channels, two (2) dial up telephone lines were used to form a single communications channel, thus giving each CC a maximum of four (4) full duplex channels. These channels could be evenly distributed among the three (3) computers in the network, giving two links between each pair of computers, or they could be distributed unevenly to match the traffic requirements. A single automatic calling 
unit was employed by each CC to automatically dial lines as required. This single unit by a special multiplex arrangement was shared among the direct dial telephone lines.

In the fall of 1973 the dial-up communication channels will be replaced with point-to-point channels operating at 4800 baud. These full duplex channels require only one line rather than the two lines previously used.

The number of logical connections between computers is a function of facilities available to service those connections themselves. These logical connections are multiplexed by the CC over the physical connections.

\section{Command Language}

A network command language exists for communicating with remote hosts. Commands exist for logging into remote hosts, copying files to and from remote hosts, transmitting and receiving records with remote hosts and a number of other useful activities. Since network access is only through a local host computer, an overhead in terms of local processor time and money is associated with communicating with remote hosts. 


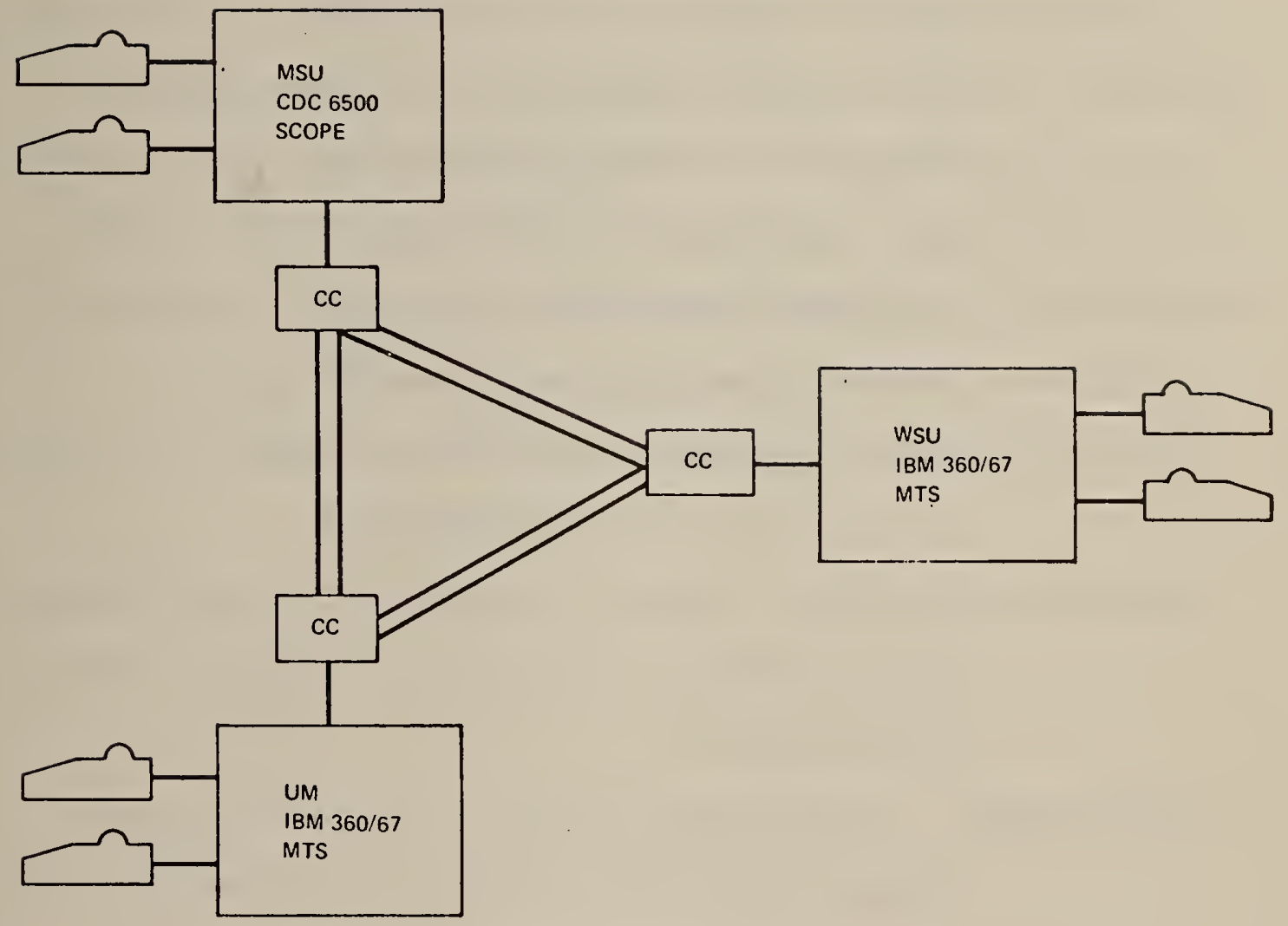

Figure 3 
Communications Circuits

Internode - circuits are voice grade, dial-up using Be11 Telephone 201A's to operate at 2000 baud. Full duplex is achieved by associating two (2) dial-up lines with each channel. Eight lines stem from each node for sending and receiving, thus forming 4 full duplex 2000 baud channels. Upgrading to point-topoint lines using Bell Telephone 208's which operate at 4800 baud. Each full duplex circuit will require one line.

Terminal to network - access to network is only through host computers. No special terminal interface exists.

Interprocess - connection between a pair of processes appears as an I/O device in each host. Once allocated to a process, a connection is dedicated to that process.

Communications Hardware

Host interface - interfaced through Communications Computer (CC) (DEC PDP-11) which can also act as a store-and-forward node. The CC appears as a number of identical but separate I/O devices to its host. 
Terminal interface - only through host computer. Communications Software

Initial connection - function is distributed and accomplished by allocating a connection device (host thinks its an I/O device).

Protocols - network interactive service allows users to access resources on a remote host and to control file transfers and process-toprocess computing. Network batch allows users to submit batch jobs on their local host for subsequent execution and/or printing elsewhere. 
Configuration

Topology

Distributed - nodes exist in three universities, one node per university.

Actual distribution - Michigan State University, University of Michigan, and Wayne State University, all in Michigan.

Composition

$$
\begin{aligned}
\text { Heterogeneous - } & \text { IBM } 360 / 67 \\
& \text { CDC } 6500
\end{aligned}
$$

Size - 3 nodes with one host computer at each. 


\section{Salient Features}

A special multiplexing system was developed to allow an automatic calling unit to be shared by eight (8) dialup 1 ines.

A network command language exists for communicating with remote computers and provides some file and record transfer capability.

Communication interface units have been designed to permit the use of wideband facilities if desired at some future time.

Host software has been greatly simplified by allowing the host to treat the communications computer as several peripheral devices. 
Interactive

Code - no terminal interface but Communications Computers translate local host character strings to ASCII.

Speed - as determined by host through which terminals interface.

Mode - as determined by host.

Remote Job Entry

Protocols - none.

Device support - as supported by host. No separate RJE network interface exists.

Minicomputer-based Systems

Interface - no special interfaces or software support other than what may be supported locally by a host computer.

Computer-to-Computer

Protocol - exists for host to host communications. Implementation - network connections appear as I/O devices to a process. 


\subsection{ARPANET}

ARPANET is a distributed network of heterogeneous computers employing store-and-forward packet-switching communications techniques. There has been interest shown in making ARPANET technology publicly available, possibly on a regulated basis. At this time there is a proposal by Packet Communications Inc, to offer a packet-switching service providing the host interfaces, network control programs, communications 1 inks, and terminal processors to compete for the data communications market [37]. This proposal stands as a formal application to the FCC along with an application by MCI Data Transmission Corporation (MDT) to offer a similar kind of service. Two other corporations, Telenet Communications Corporation and Telecommunications Network Inc., have also expressed an interest in this type of service.

\section{Overview}

The communications network or communications subnet is composed of interface message processors (IMP) and terminal IMPS (TIP) connected in a distributed fashion by 50,000 baud synchronous wide band circuits (see Figure 4). The IMPS are Honeywe11 DDP 516's with 16,000 words of memory which act as interfaces for host computer systems and as store-and-forward nodes in the network. A host is connected to an IMP and thus to the communications subnet by a channe1, and if the host is very distant from the IMP, it can con- 
nect via a synchronous communications line using block control procedures [38]. The latter method is appealing in that it eliminates the requirement of locating the IMP, a component of the communications subnet beside the host computer that wishes to connect to it. It is conceivable that the communications subnet could become physically independent and hosts would connect to the nearest IMP using the synchronous link. Presently each IMP can interface from one to four host computers to the network. The software interface between host and IMP is called the network control program (NCP). It is responsible for initiating connections, regulating traffic flow, and realizing network protocols between all communicating processes. The NCP resides wholly in the host computer thus making the host an integral part of the communications network for communications to and from the host. The NCP is closely related to the supervisor of the host system [39] and is difficult and time consuming to implement for even first rate systems personnel. Proposals have been presented to transfer some of the NCP functions to the interface processor and possibly diminish the developmental efforts of interfacing a new host [40]. In addition, some organizations are investigating the use of minicomputers as front end processors performing the NCP functions in addition to such functions as peripheral control. It is important to realize that tradeoffs do exist in the different approaches. 
Moving the NCP into a front end processor eliminates the need to make extensive alterations in the host system software and can reduce the overhead associated with running the NCP in the host computer. However, a11 communications to and from the host computer would be limited to the capabilities of the host's standard software and hardware controller.

\section{Packet Switching}

Host-to-host messages are passed from the sending host to its IMP, where they are broken into packets (thus the term "packet switching" used in describing the ARPANET) and relayed to their destination by the network of IMP's using store-and-forward techniques. Packet routing is adaptive to the conditions of the network considering failures and loading characteristics and, in general, the several packets of a single message may follow different routes. The destination IMP will reassemble the message and deliver it to proper host. The overhead of dynamic routing should be compared to the advantages gained over techniques where a route is chosen for communicating between two processes on the basis of communication facilities loading and then not dynamically altered for the duration of the connection.

\section{Protocol Leve1s}

Protocols in the network are constructed according to a layered approach. The lowest level protocol is a synchronous communications protocol governing traffic exchange between 
the IMPs. Error checking is extensive and retransmission takes place as necessary. The so-called "first level" protocol governs the exchange of information and data between host and IMP. The "second level" protocol governs the logical exchange of information between Network Control Programs in communicating hosts. The "third level" of protocol refers to any communications occurring between processes in host machines. Such protocols include the initial connection protocol, which can be used by all processes to initially communicate, the file transfer protocol, the remote job entry protocol, and the telnet protocol. Some third level protocols make use of other third level protocols. For example, the remote job entry protocol as recently defined uses the telnet protocol which in turn uses the initial connection protocol, and it also uses the file transfer protocol. The hierarchy of protocols is such that all higher level protocols use the lower level protocols to function.

\section{Termina1 Interface}

TELNET is a special third level protocol which defines a network virtual terminal and permits all terminals on the network to provide a similar interface to processes in any host. This allows for the use of any terminal supported by the network, and support is extensive, with any host system on the network even if a host could not support a particular terminal through its standard terminal controller. A special IMP which is augmented by the addition of $12 \mathrm{~K}$ of memory and 
a multi-line controller can provide direct network access to terminals (dial-ups, leased, and hardwired) without going through any host by providing the TELNET function and NCP itself [41]. This device is called the TIP. It has portal access for 63 terminals but due to buffering restrictions may be constrained to support a smaller total number when some of the terminals are operating at higher speeds. Synchronous interfaces are presently not provided. Input speed can be as high as 2400 baud asynchronous and output speed can be set up to 19200 baud under special conditions where the terminal supplies clocking. A great deal of flexibility exists to accommodate the peculiarities of different terminals. A user can specify code, speed, when to transmit (on-line feed for example), and how to echo and by which network component echoing is to be performed. Some of this flexibility is, however, accompanied by considerable cost in overhead, particularly the ability to support the variety of devices using non-standard codes. This overhead cost can be measured by the large amount of main memory reserved to facilitate these devices [2].

\section{Character Transmission}

In the servicing of interactive terminals on the ARPANET there exists a potential problem area. This occurs when terminals are interacting with a system in echo-plex mode. The echoing is often not one-to-one, that is, a particular character inputted from a terminal may require a different 
character or character string to be echoed. Also certain characters require an immediate host system reaction. Therefore the user will choose to have echoing performed by the host system rather than the TIP, which can only echo characters exactly as inputted at the terminal. This requires that packets be sent through the network containing only one character if the echoing process is to maintain pace with the input process. Since there are approximately 150 bits of overhead per transmission, then approximately 158 bits must be transmitted of which only 8 bits are data. Added to this are 158 bits of additional overhead for the echoed character transmitted from the host system. Even if characters are sent in larger blocks, six (6) at one time for example, the result will be very choppy echoing, in addition to an overwhelming overhead of approximately 700 percent. With moderately or heavily loaded network, this kind of overhead would certainly be intolerable. The solution involves putting additional sophistication in the TIP so it can echo characters as the host system would. Since the host systems are heterogeneous, there would have to be a mechanism for exchanging control information between a host and TIP to notify the TIP of idiosyncracies in echoing. Then with characters being echoed at the TIP to which a terminal is connected, it would be possible to buffer an 
entire line to form a packet for transmission on the network. This problem and potential solutions are presently receiving attention in the ARPANET community. 


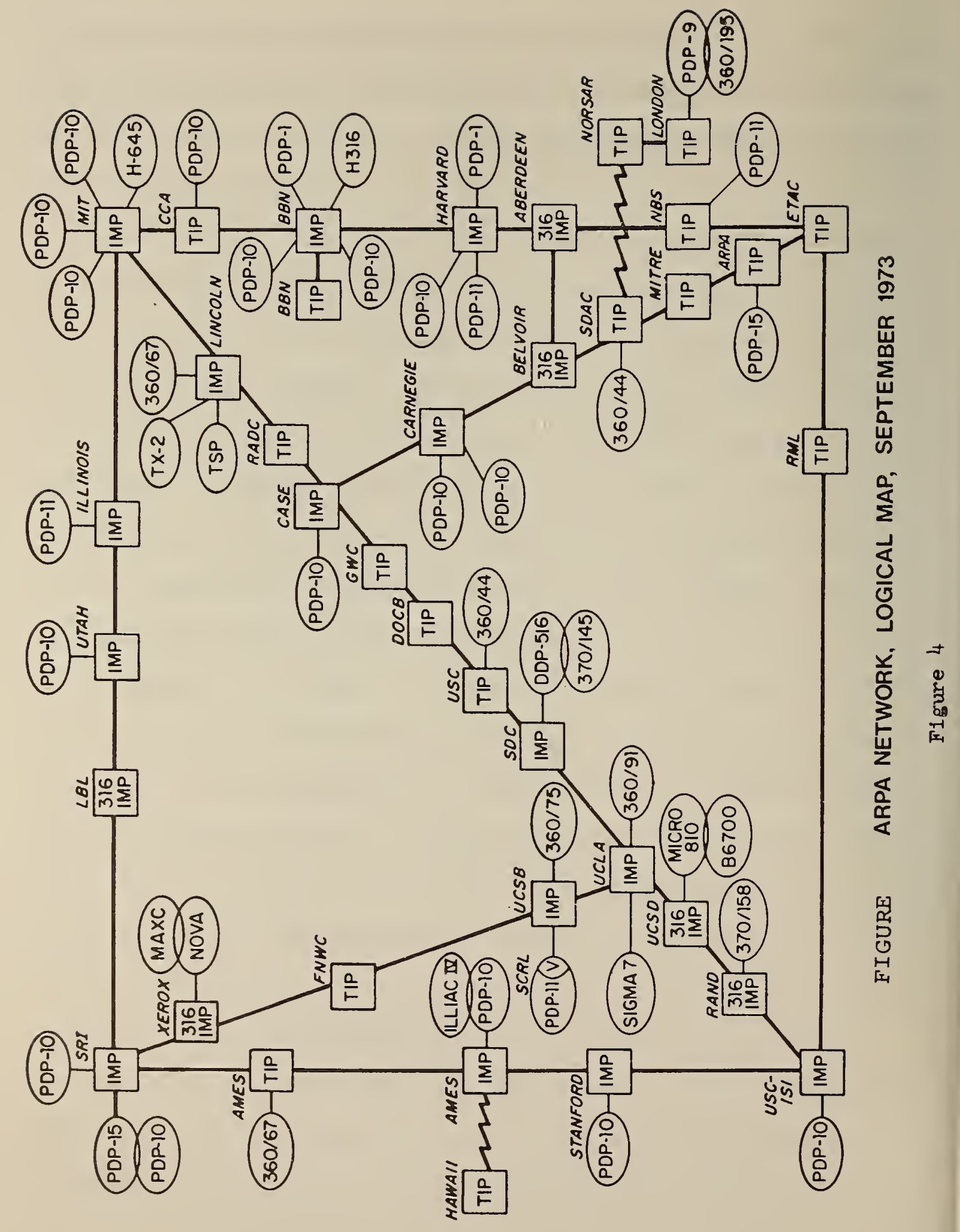




\section{Communications}

Communications Circuits

Internode - circuits are leased, full duplex, synchronous, 50,000 baud wideband. It is possible to use other speeds and 230,000 and 9600 baud have already been employed in two applications.

Terminal to network - leased or dialup, full or half duplex, asynchronous. Speeds can be from 75 to 2400 baud on input and up to 19200 baud on output.

Interprocess - packet switched at communications network leve1 (IMP to IMP).

Communications Hardware

Host interface - host is connected through the interface message processor (IMP) which also acts as a message switcher. Four hosts can be interfaced through one IMP. The IMP is a Honeywe11 DDP-516 or 316 with 16000 words of memory .

Terminal interface - terminals are interfaced through the terminal IMP (TIP) concentrator which also acts as a message switching node. Up to 63 terminals can be interfaced by one TIP (this limit is theoretical as buffering problems prevail before it is realized depending on terminals speeds). The TIP is a 
Honeywe11 H-3.16 with 28,000 words of memory.

Communications Software

Initial connection - this function is distributed and carried out by network control programs which are host resident.

Protocols - four levels of protocol exist with the highest level controlling interprocess communication. At this level exist the initial connection protocol, data and file transfer protocols, remote job entry protocol, and telnet protocol (see text of this section). 


\section{Configuration}

Topology

Distributed - hosts, IMP's, and TIP's connected in a distributed fashion.

Actual distribution - national coverage with 38 locations throughout the country, with connections to Hawaii, Norway, and England.

Composition

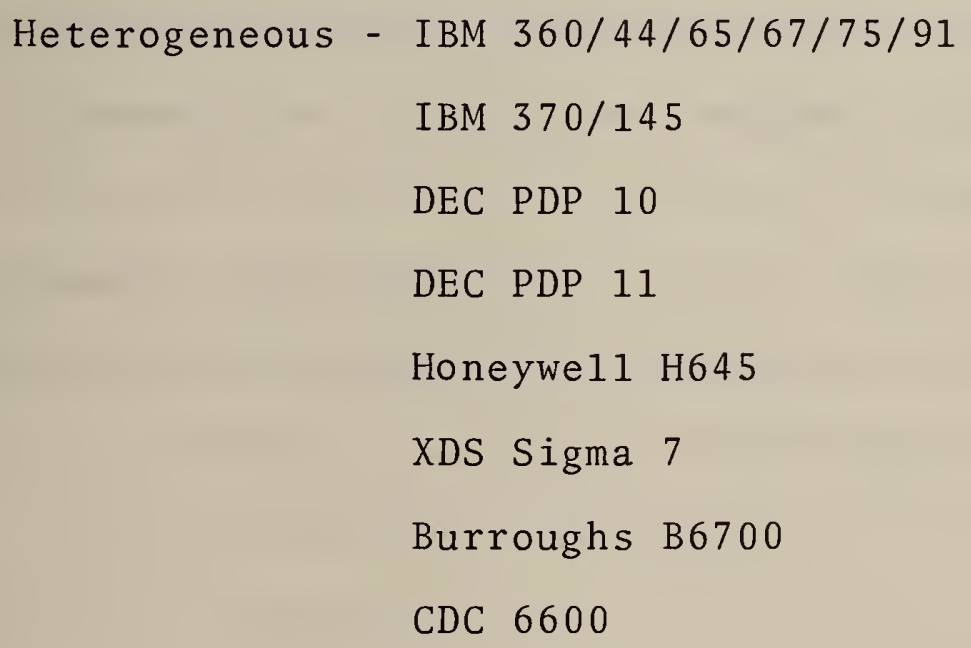

Size - approximately 40 host computers connected through 21 IMPs and 18 user nodes or TIPs (terminal users can also access network through host computers). 
Salient Features

A minihost system (ANTS) has been developed at the University of I11inois to run on a PDP-11 [22]. That minihost can be used to provide device support and user support not available through the TIP.

A telnet protocol exists which allows for the use of any terminal on any system by mapping the real terminal into the network virtual terminal.

A remote job entry protocol has recently been developed. Dynamic routing techniques are used to route messages through the network.

New versions of IMP software are loaded from a control center passing from IMP to IMP without the necessity of manual intervention at each site. 


\section{Terminal Support Capabilities}

Interactive

Code - network code is ASCII.

Non-standard terminals are mapped into ASCII at the interfaces for transmission on the network.

Speed - 75 - 2400 baud.

Mode - full-duplex with half-duplex support provided. Remote Job Entry

A Protocol exists requiring an operator using TELNET and

a terminal to initiate the various phases. There exists no special RJE device support or synchronous interface. Minicomputer-based Systems

No synchronous interface to allow for useful employment, but an NCP has been written for a minicomputer (PDP-11)

to allow it to act as a minihost.

Computer-to-Computer

Protocols exist for host-to-host communication (see text of this section). 


\subsection{TSS}

TSS is an experimental network of homogeneous computers, all presently IBM/360 model 67's, designed and implemented as a joint effort by Carnegie-Mellon University, Princeton University and IBM Research Division. The project was conceived to investigate the uses and advantages of computer networks while keeping modifications to existing hardware and software to implement that network at a minimum. The network is point-to-point, dialup, and involves no front end computers, message switching, or special interfaces for terminals (see Figure 5) .

\section{Operating System}

All of the systems are IBM $360 / 67^{\prime}$ s operating under the time-sharing system TSS/360. Systems modifications to TSS were intentionally kept at a minimum. Only the following were necessary: (1) the basic system initialization routine was modified to permit the processor to accept incoming calls from other installations; (2) the task initialization routine was modified so a task can determine whether it is operating on a primary (local) or secondary (remote) processor; (3) the central terminal communication mechanism was modified to detect communication with another location and therefore make calls to the Computer Access Method (developed for networks), rather than the customary Terminal Access Method, which in essence allows a primary process to appear as a terminal to a secondary process, and thus facilities normally available 
to terminals are at the disposal of the primary process;

(4) the task termination routines and the task abnormal termination routines were modified to transfer control to the communications logoff routine if processing is occurring on a secondary processor.

Communications Controller

The hardware used is the standard communications contoller for IBM computers, the hardwired IBM 2701 Transmission Control Unit. The unit must have a Synchronous Data Adapter and communicates using the Binary Synchronous Communications protocol. This allows for the half duplex mode of operating, that is, two-way nonsimultaneous transmission. Data flow is in one direction at a time, then the IBM 2701 control unit must reverse the 1 ine direction at the end of each exchange. The IBM 2701 controls a 2000 baud dialup modem for pointto-point switched operation. The IBM 2703 Transmission Control Unit can be substituted for the IBM 2701 in this application because transmission speeds greater than 48000 baud are not used.

\section{Computer Access Method}

The communications software, residing in the IBM $360 / 67$, which was created for the network to access the hardwired IBM 2701 or IBM 2703 Transmission Control Unit is called the Computer Access Method (CAM). CAM has the TSS standard access capabilities of open, read and write, error recovery, check (to check the completion status of the previous I/O 
operation), and close. When communication with another computer is taking place, I/O calls are made to CAM which effectively makes a processor appear as a terminal to the other processor so that processors communicate in their normal I/O stream having all the facilities normally available to a terminal at their disposal.

\section{Network Commands}

A number of network commands were developed to control communications with remote processes. One command establishes the initial communication with a remote processor. This is accomplished by dialing automatically the correct number to establish the communications link between the two independent processors. Another command can interrupt the communication with the network and temporarily return control to the TSS command interpreter. This command has a complementary command which can terminate the suspension and return control the network command interpreter to resume the previously interrupted interaction. The send data set and receive set commands are able to transfer entire files between remote processors. Finally a command exists to terminate communications with a remote processor. 


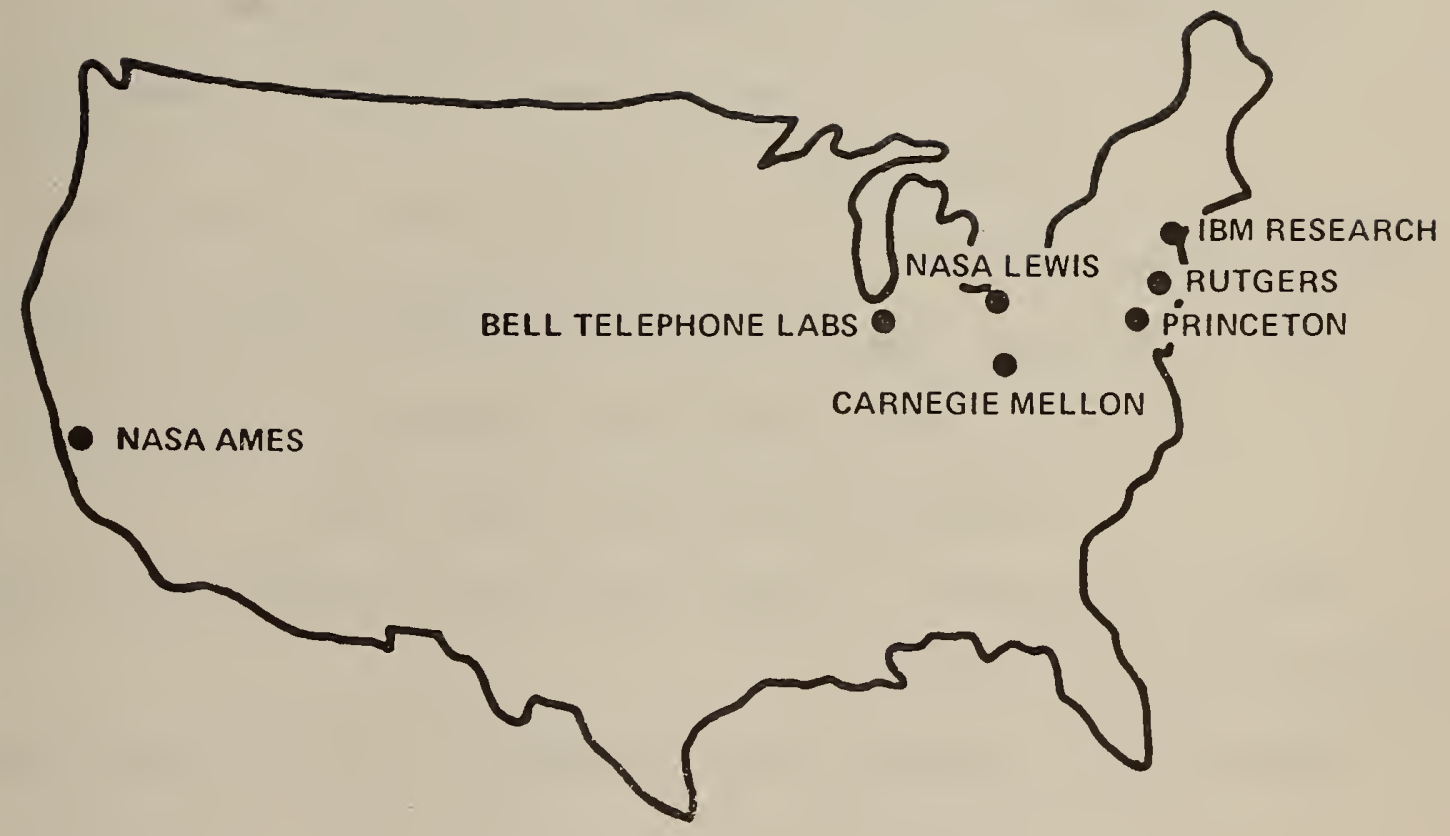

Figure 5 


\section{Communications}

Communications Circuits

Internode - 2000 baud dialup lines operating in halfduplex.

Terminal to network - no separate interface. Connection is through host computer only.

Interprocess - line switched. A switched physical circuit is devoted to each pair of communicating processes.

Communications Hardware

Host interface - host is an integral part of the communications network and connects through its standard controller (IBM 2701 ).

Terminal interface - none other than through host computer. Communications Software

Connection - Computer Access Method (CAM) residing wholly in host system establishes physical connection by dialing for 2000 baud switched circuit, and uses it for the logical connection between processes.

Protocols - simple protocols to allow for initial connection to a specific node, initiation of computation process, disconnection from a node, test outstanding responses, and send and receive data sets. 


\section{Configuration}

Topology

Distributed - hosts are distributed and communicate through dialing.

Actual distribution - Carnegie Mellon University, Princeton University, Rutgers University, IBM Research, Nasa Lewis, Nasa Ames, and Bell Telephone Labs.

Composition

Homogeneous - IBM 360/67.

Size - 7 host computers. 


\section{Salient Features}

The design philosophy of using existing communications components and the terminal look alike techniques (a processor looks like a terminal to the processor with which it communicates) enable the processors to be easily interconnected for the purpose of experimenting with networks.

A simple network control language exists enabling a user to perform some unsophisticated but useful functions. 
Interactive

Code - no terminal interface other than host computer exists.

Speed - as determined by terminal/host interface, usually

10 or 15 characters per second.

Mode - half-duplex when using standard terminal/host interface.

Remote Job Entry - no special support.

Minicomputer-based Systems - no special support.

Computer-to-Computer

Using the Computer Access Method (CAM) one computer connects as a terminal to another and vice versa allowing for interprocess communication. 


\subsection{CONCLUSIONS}

Various approaches to computer networking technology have been realized in the six networks discussed. The relative merits of the different types of components and configurations were discussed in previous chapters. It is useful now to consider briefly these network technologies relative to their potential application in the Networking for Science Program. Only the communications network, or in ARPANET terminology the "communications sub-net," will be considered. The communication network will, however, include considerations relevant to the host interface and of course the terminal interface. Furthermore, when discussing the alternatives, it is important to distinguish between a network technology and the implementation of that technology. For example, to implement a particular technology as it appears in an existing network, it might be possible to build an identical or customized version, connect to the network, or lease service from the network. These implementation alternatives are not addressed in this particular report.

\section{TSS}

The last three networks outlined were the research networks TSS, MERIT, ARPANET. TSS technology can be distinguished by a number of important features. Looking at the configuration it is distributed and homogeneous. The homogeneity certainly impedes broadening the use of TSS, and is not easily overcome as the network is composed of computers of the same model and 
same operating systems. The communications circuit and hardware are also limiting factors. The circuits are circuit switched rather than message switched which is not necessarily detrimental but is somewhat less flexible. The host interface is a non-programmable IBM $270 \mathrm{X}$ which eliminates the front end processing possiblity and thereby restricts the kinds of computers that can be interfaced. No separate terminal interface exists. The fact that there is no multiplexing of communications circuits further hinders usage. The general principle of the TSS approach, that is, having computers dial one another and communicate through their terminal handling facilities has some merit as it allows for rapid, relatively inexpensive startup. The concept would have to be modified in order to include heterogeneous systems, line multiplexing, and separate terminal interfaces.

\section{MERIT}

The MERIT network technology with its additional sophistication overcomes some of the drawbacks of the TSS approach. It does in fact include heterogeneous computers. Two different kinds of computers and three different operating systems are connected. The host interface is a Digital Equipment Corporation PDP-11 minicomputer that provides some front-end processing capability and an interface flexible enough to handle more than one kind of computer. Better circuit utilization is achieved through 1 ine multiplexing. However, there exists no direct terminal interface which implies that the minimum connection 
mechanism is through a host computer system. Furthermore, since the MERIT technology has been applied to interconnect only three computers, it has not been demonstrated that the technology could be applied unaltered to a large network of distributed computers and users.

\section{ARPANET}

The ARPANET technology appears to be the most sophisticated network technology presently in operation on a national basis. It connects heterogeneous computers of many different manufacturers in locations throughout the country. It is distributed in topology, switching, control, and computing power, making it extremely flexible for alternatives in each category. The communication circuits are message switched utilizing 50 kilobit per second wideband links. The host interface is a programmable minicomputer which as an interface is able to accommodate many different kinds of host computers including more than one simultaneously, and as a node in the network, has message-switching store-and-forward capabilities. In addition it has the ability to perform dynamic routing through the network. The terminal interface is also a minicomputer capable of supporting a large number of terminals, running in a wide range of speeds, operating in different modes, and using standard as well as non-standard character codes.

It is perhaps because of these extensive capabilities that there exists unanswered questions concerning the ARPANET technology. For instance the host interface through its 
sophistication and complexity is quite difficult to implement. It requires a great deal of time (actual amount of time and money is presently undocumented) for a systems programmer familiar with both the interface and the host operating system. This is because the network control program resides in the host computer itself. There are presently efforts underway on a limited basis, to move the network control program out of the host into a front end computer [22], and there are proposals which favor protocols for interprocess communication with less host dependency [40]. It is important to point out that a large number of NCP's have already been implemented for a variety of computer systems which could be used in newly attached identical systems. However, in the present state of the ARPANET, the network control program and its associated overhead of CPU time and core used must be considered as directly related to the communications network.

The terminal interface processor (TIP), as noted, has extensive capabilities to handle selected speeds, codes, and modes of operation. Nevertheless, there do exist shortcomings with the TIP. It can only support asynchronous terminals and in order to connect with most remote job entry devices synchronous support is needed. One way to implement synchronous support is to contain it in the already overburdened TIP. Another approach, one which has already come into existence in one instance, is the use of a minicomputer as a mini-host system on the net, providing the desired device 
support. The Center for Advance Computation at the University of Illinois has developed such a system using a DEC PDP11. The system is called the ARPANET Terminal System, or ANTS for short [22]. At any rate there needs to be a standard network-wide remote job entry device controller.

Network traffic in terms of quantities and characteristics is another potentially troublesome area. With peak loading of roughly ten percent capacity, it has never really been adequately demonstrated that the network will not deteriorate under conditions of maximum loading. Besides quantity, the characteristics of the traffic may result in poor network performance. To date most of the traffic has been that associated with terminal-to-computer interaction. It is an open question what will happen to response times when mixes of interactive (single packet) and file transfer or remote job entry (multiple packet) traffic occur. Analysis, however, has shown that success is highly probable with significantly different degrees of overhead for different traffic mixes [12].

Finally, there exists a very human problem that occurs when trying to use the network, and is likely to occur with any very heterogeneous network. With the proliferations of systems, it is cumbersome to learn the system command sequences necessary to perform even simple transactions, such as logging-in on each system. One possible solution would be to implement a higher level protocol (fourth level?) to standardize some of the basic interactions between a user and 
a system. This protocol could be realized in the hosts, TIPs, or quite conceivably on mini-hosts systems such as that under development for remote job entry devices.

\section{CYBERNET}

The commercial networks--GE, CYBERNET, TYMNET-- have developed technologies which appear to have successfully satisfied their own needs and in some cases could be useful for applicability in the Networking for Science Program. CYBERNET is a distributed network in all respects. It is heterogeneous in that it connects different models of Control Data computers. Other manufacturers' computers can connect as peripherals to the network by looking 1 ike a CDC network standard device. The look-alike technique is used quite extensively in the CYBERNET approach even for communications between host systems. The communications circuits are varied using wideband, voice grade, and public switched, but for the most part wideband circuits are used for interconnecting computers. Instead of using message-switching and store-and-forward computers, wideband switches are employed so that various interconnections are established by dialing, or having dialed, the appropriate number. The host interface to the network is the wideband switch. Apparently batch terminals can also interface to the network through the switches but normally interface directly through the host systems. Interactive terminals interface through different host systems--the CDC 6400's running the KRONOS time-sharing 
system--which can then themselves access the batch systems. The technology does not at this time seem particularly suitable as the technology for the Networking for Science Program. The use of wideband switches rather than message-switching computers limits the interconnections that can occur. No host interface other than the switch exists and no terminal interface exclusive of a host system exists.

\section{GE Information Services}

The GE approach represents an entirely different kind of network technology. It is a centralized network with all host computers interconnected but located in a central location. It is, however, possible to connect a customer computer at the periphery of the network for the purpose of exchanging files with one of the central systems. This innovation has been put into practice to make customer's batch created files available on-line to a national distribution of the customer's own users [35]. The network is heterogeneous with different models of Honeywell computers running different operating systems. Plans call for connecting to the network computers from other manufacturers [34]. Also the concept of allowing customers to connect their own computers to exchange files with the central system and utilize the vast communications network is planned to be expanded.

The communications circuits are message-switched with the apparent restriction that communications are with one of the central systems. The host interface is a computer, but 
only systems at the central site are integral to the network. As mentioned, however, certain customer computers can interface as remote job entry devices. Communications are directed to the central systems so that if many customer computers are connected to the network it is not clear that they could establish direct communications with one another using the GE communications network. Also terminals connected to the network through the terminal interfaces communicate with the central system and may not be able to communicate with customer computers. This centralization is the drawback to use of the technology per se in a distributed network although the extensive coverage and capabilities of the net may warrant further consideration.

It is useful to point out that the technology itself may be applicable to networks of distributed computers in two different ways. First, the central complex may be viewed as a large message switching center. A11 communications between computers connected to the remote concentrators at the periphery and customer's terminals could be routed through the central switch. Second, the technology used to interconnect the computers at the central complex could be applied to a network of distributed computers. Both of these are conjecture. TYMNET

TYMNET has already entered the business of offering only the services of the communications network exclusive of the Tymshare host computers. In such cases customers interface their own 
host computers and their users, their own terminals, and thereby utilize the TYMNET national data communications network. This reportedly is permitted under Federal Communications Commission Tariff 260 covering joint use agreements. The customers make a contractual arrangement with Tymshare for use of a certain percentage of the capacity of TYMNET for handing the communications traffic between the customer's terminals and customer's host system or systems. The Telephone Company then bills the customer for the specified percentage of the communications charges. In addition, Tymshare bills the customer for value added services. These include the utility of the host and terminal interfaces along with the store-and-forward capability of the communications computers. Also included is the involvement of the Tymshare host computer running the supervisor which establishes all initial connections.

The TYMNET technology has a number of characteristics that could make its usage a powerful convenience. It has been demonstrated that it can support a network, well distributed, with nodes, computers, and users all over the nation and in Europe with a clear possiblity of connecting new host systems and users wherever they may presently exist. The host computers themselves are heterogeneous being different models and from different manufacturers and it appears conceivable to connect computers of any of the primary manufacturers using their standard interface equipment. The communications 
circuitry is voice grade connecting the switching nodes which are store-and-forward minicomputers. The voice grade circuits appear to be quite sufficient for the interactive traffic that exists on the network and some rather impressive figures exist to indicate a high usage of the facilities through efficiencies in line concentration. For example forty-six (46) interactive users are supported on a 2400 baud facility.

Switching is performed by establishing a virtual circuit through the nodes of the network. The systems supervisor, the system initially contacted by every user, sends information to the nodes to set up a virtual circuit from user process (terminal) to server process (computer). This virtual circuit concept would seem to decrease communications overhead once the circuit is established, although there is the disadvantage that dynamic re-routing cannot occur to accommodate circuit failures. Nevertheless, the virtual circuit reportedly results in nearly an 80 percent efficiency in line utilization rather than the 60 percent efficiency associated with true message switching [7]. This is due primarily to a reduction in overhead due to the use of only a single character to address a virtual channel. Furthermore, the central supervisor performing all connections presents an advantage: only the supervisor has global knowledge of the network so that the individual nodes can be handled independently relative to software changes and debugging. In the event of a failure at the supervisor node, a backup system will take 
over, first polling the network to determine its status.

The host interface to the network is a minicomputer, which for the sake of convenience, can make the network appear as terminals to the host's regular terminal interface, although the interface is more sophisticated in the case of Tymshare host computers. Interfacing new computers in the past has not been particularly difficult. The terminal interface is also a minicomputer acting as a concentrator and a storeand-forward node. It can handle terminals of a variety of speeds and when communicating with some of the host sytems will perform local echoing.

The network technology appears quite capable of satisfying the communications needs of a network of computers distributed throughout the country, and large numbers of interactive users (presently 900 simultaneously). With a present average number of failures of 1.4 per year per node, reliability is another asset. One big drawback to the network is its lack of support of synchronous devices and remote job entry. This situation may not be permanent as plans call for the implementation of synchronous device support to control certain types of remote job entry devices. Also batch processing is anticipated on the Digital Equipment Corporation PDP-10 computers which have become a part of the network. Also the incorporation of large IBM computers into the system is underway for more remote job entry and batch processing. 
In keeping with the purposes of this report, the discussion has been exclusive of economic considerations, concentrating on functional capabilities. All of the networks discussed have particularly worthy features. It is, therefore, worthwhile to suggest at this time that a single network technology need not be selected. It is entirely possible to use the best features of each network technology matching the task to the proper network.

Although the discussion of the usage of services provided by the host computers of the networks did not fit into the context of this report, it is important to the concept of multiple networks. Suffice it to say that if the services are readily available and compatible with the needs of user groups, than the most convenient access may be through the networks to which the hosts are already attached.

Two of the networks discussed--TYMNET and ARPANET--are presently offering a computer communications service to which a customer can connect computers acting as host systems, as well as terminals. Each has considerable experience in host computer and terminal interfacing and is representative of a computer communications network to which customers can connect their servers and users, nationally distributed, with a minimal effort in communications development. Both networks, resultant of both their present state of development and the inherent characteristics of their technologies, represent important 
advantages and also possess serious limitations, the details of which are described in the text of this report. 
1. Aufenkamp, D. D., "NSF Network Initiative," Networks and Disciplines, EDUCOM, Princeton, N.J., 197 $\overline{3, \text { p. 88- }}$ $\overline{90 .}$

2. Pyke, T. N. and R. P. Blanc, "Computer Networking Technology--A State of the Art Review," Computer, IEEE Computer Society, August 1973, p. 13-19.

3. Blanc, R. P., I. W. Cotton, T. N. Pyke, S. W. Watkins, "Annotated Bibliography of the Literature on Resource Sharing Computer Networks," National Bureau of Standards Specia1 Publication 384, September 1973.

4. Abrams, M., G. Lindamood, T. Pyke, "Measuring and Mode1ing Man-Computer Interaction," Proc. First Annual ACM SIGME Symposium on Measurement and Evaluation, Palo A1to, California, February 1973, p. 136-142.

5. Jackson, P. and C. Stubbs, "A Study of Multi-access Computer Communications," Proc. SJCC 1969, p. 491-504.

6. Fuchs, E. and P. Jackson, "Estimates of Distributions of Random Variables for Certain Computer Communications Traffic Models," CACM, Vo1. 13, No. 12, December 1972, p. $752-757$.

7. Schwartz, M., R. Boorstyn, R. Pickholtz, "Termina1Oriented Computer Communications Networks," Proc. IEEE Nov. 1972, p. 1408-1422.

8. Chu, W. W., "A Study of Asynchronous Time Division Multiplexing for Time-Sharing Computer Systems," Proc. SJCC 1969, p. 669-678.

9. Tymes, L., "TYMNET--A Terminal Oriented Communication Network," Proc. SJCC 1971, p. 211-216.

10. Luther, W., "Conceptual Bases of Cybernet," Computer Networks, ed. by Rustin, Randal. Prentice-Ha11, Inc., New Jersey 1972, p. 111-146.

11. Hench, R. and D. Foster, "Toward an Inclusive Information Network," Proc. FJCC 1972, p. 1235-1242.

12. "Interface Message Processors for the ARPA Computer Network," Bolt, Beranek, and Newman Quarterly Technical Report No. 16, Oct.-Dec. 1972.

13. Abramson, N., "Packet Switching with Satellites," U. of Hawaii Technical Report B73-2, March 1973. Also 
paper in Proc. NCC 1973.

14. Institute for Computer Research Quarterly Report No. 34, Aug. 1972. University of Chicago.

15. Blanc, R., "Minicomputer Trends and Applications--1973," Computer, IEEE Computer Society, June 1973.

16. Pyke, T., "Some Technical Considerations for Improved Service to Computer Network Users," COMPCON 73 - International Conference of the IEEE Computer Society, Feb. 1973, p. $53-56$.

17. Thomas, R. and D. Henderson, "McROSS-A Multi-computer Programming System," Proc. SJCC 1972, p. 281-293.

18. Frank, H., "Research in Store and Forward Computer Networks," Network Analysis Corporation, Glen Cove, N.Y., December 1971.

19. Heart, F., R. Kahn, S. Omstein, W. Crowther, D. Walden, "The Interface Message Processor for the ARPA Computer Network," Proc. SJCC 1970, P. 551-567.

20. Beere, M. P. and N. Sullivan, "TYMNET--A Serendipitous Evolution," Trans. IEEE, Vo1. COM-20, No. 3, June 1972, p. $511-515$.

21. McKay, D. and D. Karp, "Network 440--IBM Research Computer Sciences Department Computer Network," IBM Corporation, Yorktown Heights, N.Y., July 1971.

22. Bouknight, W. J. and S. Denenberg, "ANTS--A New Approach to Accessing the ARPA Network," U. of Illinois Report CAC No. 47, July 1972.

23. Ornstein, S., F. Heart, W. Crowther, H. Rising, S. Russe11, A. Miche1, "The Terminal IMP for the ARPA Computer Network," Proc. SJCC 1972, p. 243-254.

24. Aupperle, E., "Merit Computer Network: Hardware Considerations," Computer Networks, ed. by Randall, Rustin, Prentice-Ha11, Inc., New Jersey 1972, p. 49-63.

25. Rutledge, R., et a1., "An Interactive Network of TimeShared Computers," Proc. 24th National Conference ACM, 1969, p. $431-441$.

26. Roberts, L. and B. Wessler, "Computer Network Development to Achieve Resource Sharing." Proc. SJCC 1970, p.543-549. 
27. Crocker, S., J. Heafner, R. Metcalfe, and J. Pastel, "Function-Oriented Protocls for the ARPA Computer Network," Proc. SJCC 1972.

28. Carr, S., S. Crocker, and V. Cerf, "Host-Host Communication Protocol in the ARPA Network," Proc. SJCC 1970, p. 589-598.

29. Gray, J. P., "Line Control Procedures," Proc. IEEE, Vo1. 60, No. 11, Nov. 1972, p. 1301-1312.

30. Frank, H. and W. Chou, "Topological Optimization of Computer Networks," Proc. IEEE, Vo1. 60, No. 11, Nov. 1972, p. 1385-1397.

31. Kleinrock, L., "Computer Network Research," Quarterly Progress Report, UCLA, July-Dec. 1971.

32. Cole, G. D., "Performance Measurements on the ARPA Computer Network," Proc. ACM/IEEE 2nd Symposium on Problems in Optimization of Data Communications Systems, Oct. 1971.

33. Farber, D. J., "Data Ring Oriented Computer Networks," Computer Networks, ed. R. Rustin, Prentice-Ha11, Englewood C1iffs, N.J., 1972, p. 79-93.

34. Feeney, G., "The Future of Computer Utilities," Computer Communications: Impacts and Implementation, ed. by Winkler, Stanley, International Conferences on Computer Communication, 1972, ICCC, p. 237-239.

35. Gaines, E. and J. Taplin, "The Emergence of National Networks Remote Computing - Year VI," Telecommunications, Vo1. 5, Issue 12, December 1972, p. 27-29.44-49.

36. Herzog, B., "Merit Computer Network," Computer Networks, ed. by Rustin, Randa1l, Prentice-Ha11, Inc., New Jersey 1972 , p. $45-48$.

37. Application to the Federal Communications Commission by Packet Communications, Inc., FCC No. P-C-8533, Jan. 1973.

38. "Interface Message Processors for the ARPA Computer Network," Bolt, Beranek, and Newman Quarterly Tech. Report No. 12 , Oct.-Dec. 1971.

39. Metcalfe, R. M., "Strategies for Operating Systems in Computer Networks;" 1972 Proc. of the ACM, p. 278-281.

40. Walden, D., "A System for Interprocess Communication in a Resource Sharing Computer Network, "CACM, Vo1. 15, Issue 4, Apri1 1972, p. $221-230$. 
41. Kahn, R. E., "Terminal Access to the ARPA Computer Network," $\frac{\text { Computer Networks }}{972, \text { p. } 49-63 \text {. }}$. R. Rustin, Prentice-Ha11, 
U.S. DEPT. OF COMM.

BIBLIOGRAPHIC DATA SHEET

4. TITLE AND SUBTITLE

Review of Computer Networking Technology
PUBLICATION OR RE PORT NO. NBS TN-804
2. Gov't Accession No.

3. Recipient's Accession No.

5. Publication Date

January 1974

6. Performing Organization Code

8. Performing Organization

Robert P. Blanc

10. Project/Task/Work Unit No. 6502372

NAT IONAL BUREAU OF ST AND ARDS

DEPARTMENT OF COMMERCE

WASHINGTON, D.C. 20234

11. Contract/Grant No.

$A G-350$

13. Type of Report \& Period Covered Interim

Fourth Qtr. 1973

National Science Foundation

Washington, D.C. 20550

14. Sponsoring Agency Code

16. ABSTRACT (A 200-word or less factual summary of most significant information. If document includes a significant bibliography or literature survey, mention it here.)

This report gives a descriptive summary of the technical characteristics of existing computer networks, including data communication technology and configuration related to support of resource sharing services for a computer network. Included are discussions of terminal support capabilities for the communications network and a development of relevant network terminology. The report concludes with a comparative evaluation of existing technological approaches to networking.

17. KEY WORDS (Alphabetical order, separated by semicolons) Computer networks; computer-to-computer transfers; interactive terminals; minicomputer-based systems; network configuration; remote job entry; resource sharing.

18. AVAILABILITY STATEMENT

X] UNLIMITED.

FOR OFFICIAL DISTRIBUTION. DO NOT RELEASE TO NTIS.

\begin{tabular}{|l|c|}
\hline $\begin{array}{l}\text { 19. SECURITY CLASS } \\
\text { (THIS REPORT) }\end{array}$ & 21. NO. OF PAGES \\
UNCL ASSIFIED & 135 \\
\hline $\begin{array}{l}\text { 20. SECURITY CLASS } \\
\text { (THIS PAGE) }\end{array}$ & $\begin{array}{c}\text { 22. Price } \\
\$ 1.55\end{array}$ \\
UNCL ASSIFIED
\end{tabular}





\section{NBS TECHNICAL PUBLICATIONS}

PERIODICALS

JOURNAL OF RESEARCH reports National Burcau of Standards research and development in physics, mathcmatics, and chemistry. Comprehensive scientific papers give complete details of the work, including laboratory data, experimental procedures, and theoretical and mathematical analyses. Illustrated with photographs, drawings, and charts. Includes listings of other NBS papers as issued.

Published in two sections, available separately:

\section{- Physies and Chemistry (Section A)}

Papers of interest primarily to scientists working in these fields. This section covers a broad range of physical and chemical research, with major emphasis on standards of physical measurement, fundamental constants, and properties of matter. Issued six times a year. Annual subscription: Domestic, $\$ 17.00$; Foreign, \$21.25.

\section{- Mathematical Sciences (Section B)}

Studies and compilations designed mainly for the mathematician and theoretical physicist. Topics in mathematical statistics, theory of experiment design, numerical analysis, theoretical physics and chemistry, logical design and programming of computers and computer systems. Short numerical tables. Issued quarterly. Annual subscription: Domestic, $\$ 9.00$; Foreign, $\$ 11.25$.

\section{DIMENSIONS, NBS}

The best single source of information concerning the Bureau's measurement, research, developmental, cooperative, and publication activities, this monthly publication is designed for the layman and also for the industry-oriented individual whose daily work involves intimate contact with science and technology - for engineers, chemists, physicists, research managers, product-development managers, and company executives. Annual subscription: Domestic, $\$ 6.50$; Foreign, $\$ 8.25$.

\section{MONPERIODICALS}

Applied Mathematics Series. Mathematical tables, manuals, and studies.

Building Science Series. Research results, test methods, and performance criteria of building matcrials, components, systems, and structures.

Handbooks. Recommended codes of engineering and industrial practice (including safety codes) developed in cooperation with interested industries, professional organizations, and regulatory bodies.

Special Publications. Proceedings of NBS conferences, bibliographies, annual reports, wall charts, pamphlets, etc.

Monographs. Major contributions to the technical literature on various subjects related to the Bureau's scientific and technical activities.

National Standard Reference Data Series. NSRDS provides quantitative data on the physical and chemical properties of materials, compiled from the world's literature and critically evaluated.

Product Standards. Provide requirements for sizes, types, quality, and methods for testing various industrial products. These standards are developed cooperatively with interested Government and industry groups and provide the basis for common understanding of product characteristics for both buyers and sellers. Their use is voluntary.

Technical Notes. This series consists of communications and rcports (covering both other-agency and NBS-sponsored work) of limited or transitory interest.

Federal Information Processing Standards Publications. This serics is the official publication within the Federal Government for information on standards adopted and promulgated under the Public Law 89-306, and Bureau of the Budget Circular A-86 entitled, Standardization of Data Elements and Codes in Data Systems.

Consumer Information Series. Practical information, based on NBS research and experience, covering areas of interest to the consumer. Easily understandable language and illustrations provide useful background knowledge for shopping in today's technological marketplacc.

\section{BIBLIOGRAPHIC SUBSCRIPTION SERVICES}

The following current-awareness and literature-survey bibliographies are issued periodically by the Bureau :

Cryogenic Data Center Current Awareness Service (Publications and Reports of Interest in Cryogenics). A literature survey issued weekly. Annual subscription: Domestic, $\$ 20.00$; foreign, $\$ 25.00$.

Liquefied Natural Gas. A literature survey issued quarterly. Annual subscription: $\$ 20.00$.

Superconducting Devices and Materials. A literature survey issued quarterly. Annual subscription: $\$ 20.00$. Send subscription orders and remittances for the preceding bibliographic services to the U.S. Department of Commerce, National Technical Information Service, Springfield, Va. 22151.

Electromagnetic Metrology Current Awareness Service (Abstracts of Selected Articles on Measurement Techniques and Standards of Electromagnetic Quantities from D-C to Millimeter-Wave Frequencies). Issued monthly. Annual subscription: $\$ 100.00$ (Special rates for multi-subscriptions). Send subscription order and remittance to the Electromagnetic Metrology Information Center, Electromagnetics Division, National Bureau of Standards, Boulder, Colo. 80302.

Order NBS publications (except Bibliographic Subscription Services) from: Superintendent of Documents, Government Printing Office, Washington, D.C. 20402. 
nal Bureau of Standards

ton, D.C. 20234

BUSINESS

POSTAGE AND FEES PAID

U.S. DEPARTMENT OF CDMMERCE

COM-215

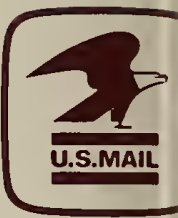

for Private Use, $\$ 300$ 



